\title{
Problems in dynamical systems and related topics
}

\author{
BORIS HASSELBLATT
}

\section{CONTENTS}

1. Introduction 274

2. Smooth realization of measure-preserving maps (Anatole Katok) 274

3. Coexistence of KAM circles and positive entropy in area-preserving twist maps (presented by Anatole Katok)

4. Orbit growth in polygonal billiards (Anatole Katok) 277

5. Flat surfaces and polygonal billiards (presented by Anton Zorich) 278

6. Symbolic extensions (Michael Boyle and Sheldon Newhouse) 278

7. Measures of maximal entropy (presented by Sheldon Newhouse) 283

8. Properties of the measure-theoretic entropy of Sinai-Ruelle-Bowen measures of hyperbolic attractors (contributed by Miaohua Jiang) 284

9. Sinai-Ruelle-Bowen measures and natural measures (presented by Michał Misiurewicz) 284

10. Billiards (Domokos Szasz) 285

11. Stable ergodicity (with Keith Burns) 286

12. Mixing in Anosov flows (Michael Field) 288

13. The structure of hyperbolic sets (contributed by Todd Fisher) 289

14. The dynamics of geodesic flows (presented by Gerhard Knieper) 291

15. Averaging (Yuri Kifer) 292

16. Classifying Anosov diffeomorphisms and actions (presented by Anatole Katok and Ralf Spatzier)

17. Invariant measures for hyperbolic actions of higher-rank abelian groups (Anatole Katok)

18. Rigidity of higher-rank abelian actions (presented by Danijela Damjanovic) 298

19. Local rigidity of actions (presented by David Fisher) 299

20. Smooth and geometric rigidity 300

21. Quantitative symplectic geometry (Helmut Hofer) 312

22. Hilbert's sixteenth problem (presented by Yulij Ilyashenko) 314

23. Foliations (presented by Steven Hurder) 314

24. "Fat" self-similar sets (Mark Pollicott) 315

References 316 


\section{Introduction}

At the Clay Mathematics Institute/Mathematical Sciences Research Institute Workshop on "Recent Progress in Dynamics" in September-October 2004 the speakers and participants were asked to state open problems in their field of research, and much of this problem list resulted from these contributions. Thanks are due, therefore, to the Clay Mathematics Institute and the the Mathematical Sciences Research Institute for generously supporting and hosting this workshop, and to the speakers, who graciously responded to the suggestion that open problems be stated whenever possible, and who in many cases kindly corrected or expanded the renditions here of the problems that they had posed. It is my hope that this list will contribute to the impact that the workshop has already had. It was helpful to this endeavor and is a service to the community that most lectures from the workshop can be viewed as streaming video at http:// www.msri.org/calendar/workshops/WorkshopInfo/267/show_workshop.

In this list, almost all sections are based on an original version written by myself about the problems as presented by the proposer in a talk during the workshop. The proposer is identified by the attribution "(presented by...)" in the section heading. Where the proposer undertook significant modification of this original version, the section became attributed to the proposer (without "presented by"). Section 8 and Section 13 were contributed by their authors without any preliminary draft by myself and were only slightly edited by me, and Section 11 is based in good part on the questions raised by Keith Burns in his talk but was written collaboratively. Finally, the collection of problems I describe in Section 20 was not as such presented at the workshop, but includes problems familiar to many participants.

\section{Smooth realization of measure-preserving maps (Anatole Katok)}

QUESTION 2.1. Given an ergodic measure-preserving transformation $T$ of a Lebesgue space $X$ with probability measure $\mu$, under which conditions is there a diffeomorphism $f$ of a compact manifold $M$ that preserves a smooth volume $v$ for which $(f, v)$ is measurably isomorphic to $(T, \mu)$ ? In particular, is there Changed "transformations" to "maps" to allow table of any $T$ with finite $\mu$-entropy for which there is no such $f$ ?

Put differently, is finiteness of entropy (which, as shown first by Kushnirenko, holds for diffeomorphisms of manifolds with respect to any invariant Borel probability measure, see e.g., [82, Corollary 3.2.10]) the only restriction imposed on smooth models of measure-preserving transformations?

A potentially useful method is that of Anosov and Katok [2] (see also [34] for a modern exposition) which provides nonstandard smooth realizations of certain dynamical systems. An important pertinent result is due to Pesin: For 
a smooth dynamical system on a surface with positive entropy, weak mixing implies Bernoulli [120]. Thus there are restrictions on smooth realizations on particular types of manifolds.

It is expected that there are indeed restrictions on realizability other than finiteness of entropy, so long as one considers smooth measures. (Lind and Thouvenot [98] showed that every finite-entropy measure-preserving transformation can be realized as an automorphism of the 2-torus with respect to a suitable invariant Borel probability measure.) Here the picture may be different for infinite versus finite smoothness. In order to establish the existence of such restrictions one needs to construct some suitable invariants. Again, on one hand one may look at specific manifolds or dimensions, such as in Pesin's aforementioned result for maps with positive entropy. For zero entropy an interesting observation is Herman's "Last Geometric Theorem" [33]:

THEOREM 2.2. An area-preserving $C^{\infty}$ diffeomorphism $f$ of the disk that has Diophantine rotation number on the boundary has a collection of invariant circles accumulating on the boundary.

The Anosov-Katok construction provides examples of nonstandard realization of rotations with Liouvillian rotation numbers. In particular, given any Liouvillian rotation number $\rho$, Fayad, Saprykina and Windsor ([36], using the methods of [35]) constructed an area-preserving $C^{\infty}$ diffeomorphism of the disc that acts as the rotation by $\rho$ on the boundary and is measurably isomorphic to it.

It should be mentioned here in passing that no nonstandard smooth realizations of Diophantine rotations are known:

QUESTION 2.3. Given an ergodic Diophantine rotation, is there an ergodic volume-preserving diffeomorphism on a manifold of dimension greater than 1 that is measurably conjugate to the rotation?

Herman's Theorem 2.2 suggests that this would be very hard to achieve on a disk.

There are several systems where existing methods might help decide whether a nonstandard smooth realization exists, such as Gaussian systems, some interval exchanges, and maybe the horocycle flow on the modular surface.

\section{Coexistence of KAM circles and positive entropy in area-preserving twist maps (presented by Anatole Katok)}

Consider the standard (twist) map

$$
f_{\lambda}(x, y):=(x+y, y+\lambda \sin 2 \pi(x+y))
$$

of the cylinder (or annulus) $C:=S^{1} \times \mathbb{R}$, which preserves area. 
QUESTION 3.1. Is the measure-theoretic entropy $h_{\text {area }}\left(f_{\lambda}\right)$ positive (with respect to area as the invariant Borel probability measure)

(1) for small $\lambda>0$ ?

(2) for some $\lambda>0$ if the problem is considered instead on $\mathbb{T}^{2}=S^{1} \times S^{1}$ (to provide an invariant Borel probability measure)?

Positive entropy implies the existence of ergodic components of positive area by a theorem of Pesin [120]. It is generally believed that the answers should be positive.

As to the first part of this question, the KAM theorem is clearly a pertinent issue: For small $\lambda>0$ a large portion of the area of the cylinder is the union of invariant circles. Nevertheless, the complement consists of regions of instability that give rise to positive topological entropy due to heteroclinic tangles associated with hyperbolic periodic points. (In higher dimension the invariant tori don't even separate these regions of complicated dynamics.) The horseshoes due to these tangles have zero measure, however, and everything one can prove using estimates of hyperbolicity type is necessarily confined to sets of measure zero. To establish positive measure-theoretic entropy, by contrast, requires control on a set of positive measure. In particular, one must ensure that the invariant circles of all scales do not fill a set of full measure. This set is easily seen to be closed, and it has a Cantor structure. Unfortunately the boundary circles are not among those obtained by the KAM theorem (this was apparently first observed by Herman), and they are generally believed to be nonsmooth, which suggests that proving this to be the boundary of the hyperbolic domain will be difficult indeed; no imaginable technique can be expected to serve the purpose.

This illustrates a fundamental problem: Just as Kolmogorov discovered the essential tools for describing complicated dynamics, the KAM theorem established, as illustrated in this essential example, that the applicability of these tools even to mechanical problems faces fundamental limitations.

As to the second part of the question, it is known that near $\lambda=0.98 \cdot 2 \pi$ the last KAM circles disappear, so one might hope for the problem to become tractable. However the elliptic periodic points don't disappear at that stage. There is a plausible scheme to make all elliptic points disappear for certain large parameter values which circumvents the global constraints of index theory by creating orientation-reversed hyperbolic points and is inspired by Jakobson's parameter-exclusion method for 1-dimensional maps[70]. This is aimed at finding parameters for which useful estimates can be carried out.

A "realistic" variant of this problem might be to consider random perturbations of this system. This is not devoid of difficulties, but might be more tractable. 


\section{Orbit growth in polygonal billiards (Anatole Katok)}

Consider the billiard system in a triangle or, more generally, a polygon $P \subset$ $\mathbb{R}^{2}$. This is an area-preserving dynamical system. The challenge is to understand the global complexity of such a system. For example, let $S(T)$ be the number of orbits of length at most $T$ that begin and end in vertices.

PROBLEM 4.1. Find upper and lower bounds for $S(T)$.

QUESTION 4.2. Is there a periodic orbit for every choice of $P$ ?

This is open even for most obtuse triangles; R. Schwartz has shown however that if the maximal angle in a triangle is less than $100^{\circ}$ then periodic orbits always exist [129].

PROBLEM 4.3. Find conditions for ergodicity of the billiard flow with respect to Liouville measure (area). In particular, is the billiard flow ergodic for almost every $P$ ?

Boshernitzan and Katok observed that based on the work of Kerckhoff-MasurSmillie [88], a Baire category argument produces a dense $G_{\delta}$ of ergodic polygons. Vorobets [134] improved this by giving an explicit sufficient condition for ergodicity in terms of the speed of simultaneous approximation of all angles mod $\pi$ by rationals. Existence of even a single ergodic example with Diophantine angles remains an open and probably very hard question

Katok [80] showed that $T^{-1} \log S(T) \rightarrow 0$, which is far from effective. For rational polygons Masur [105] showed that there are positive constants $C_{1}$ and $C_{2}$ such that $C_{1} T^{2} \leq S(T) \leq C_{2} T^{2}$. For some examples, (e.g., those leading to Veech surfaces [67]) existence of quadratic multiplicative asymptotics has been shown and even the constant calculated.

Any effective subexponential estimate (such as $e^{-T^{3 / 4}}$, say) for arbitrary polygons would be a major advance.

CONJECTURe 4.4. $\lim _{T \rightarrow \infty} S(T) / T^{2+\varepsilon}=0$ for every polygon and every $\varepsilon>0$, but $S(T) / T^{2}$ is often irregular and unbounded.

It should be said that understanding orbit growth in measure-theoretic terms with respect to the Liouville measure is not a difficult matter; one can calculate slow entropy and gets a quadratic growth rate. Indeed, Mañé observed that the number of connections of length up to $T$ between two randomly chosen boundary points is on average quadratic in $T$, i.e., statistically one sees quadratic orbit growth. Accordingly, any deviation from quadratic orbit growth would be connected to different invariant measures.

The basic problem is the lack of structure here, except for rational polygons where one can represent the problem in terms of a Riemann surface with a 
quadratic differential and then bring tools of Teichmüller theory to bear. For irrational polygons one could try to associate a Riemann surface of infinite genus in an analogous fashion, but this sacrifices recurrence. There are some borderline cases where one can use recurrence in dynamical systems that preserve an infinite measure.

The basic problem related to this circle of questions is that in these parabolic systems dynamical complexity arises from a combination of stretching and cutting. The stretching is well understood for polygonal billiards, and produces quadratic growth (geometrically a shear), but usually produces no periodic orbits; the interesting phenomena arise from cutting, which is poorly understood beyond the fact that growth is subexponential.

\section{Flat surfaces and polygonal billiards (presented by Anton Zorich)}

This topic is closely related with the previous one. In fact, by unfolding, rational billiards produce flat surfaces of a special kind. Powerful methods based on the study of the Teichmüller geodesic flow on various strata of quadratic differentials usually are not directly applicable to billiards.

QUESTION 5.1. Is the geodesic flow on a generic flat sphere with 3 singularities ergodic?

QUESTION 5.2. Is there a closed regular geodesic?

Two copies of a triangle with boundaries identified gives such a space, so this problem is related to polygonal billiards. For some rational triangles the initial direction is preserved and thus provides a first integral.

QUESTION 5.3. Is there a precise quadratic asymptotic for the growth of closed geodesics on every genus 2 flat surface?

There is much recent progress (such as the classification by Calta and McMullen of Veech surfaces in $H(2)$ - genus 2 with a single conical singularity [20;106]) to put this question into reach.

\section{Symbolic extensions (Michael Boyle and Sheldon Newhouse)}

Briefly, the effort to understand possible symbolic dynamics for a general topological dynamical system leads to the Downarowicz theory of "entropy structure", a master entropy invariant which provides a refined and precise structure for describing the emergence of chaos on refining scales. This leads to problems of the compatibility of entropy structure with varying degrees of smoothness. 
In the remainder of this section all homeomorphisms are assumed to have finite topological entropy and to be defined on compact metrizable spaces. If $g$ is the restriction of some full shift on a finite alphabet to a closed shift-invariant subsystem $Y$, then $(Y, g)$ is said to be a subshift.

DEFINITION 6.1. Given a homeomorphism $f$ of a compact metrizable space $X$ with finite topological entropy, a symbolic extension of $(X, f)$ is a continuous surjection $\varphi: Y \rightarrow X$ such that $f \circ \varphi=\varphi \circ g$ and $(Y, g)$ is a subshift.

Given $\varphi$ as above, we may also refer to the subshift $(Y, g)$ as a symbolic extension of $(X, f)$. A coding of a hyperbolic dynamical system by a topological Markov shift provides the classical example. In general, the subshift $(Y, g)$ is required to be a subsystem of some full shift on a finite set of symbols, but it need not be a Markov shift and its topological entropy (though finite) need not equal that of $f$.

DEFINITION 6.2. The topological symbolic extension entropy of $f$ is

$$
\mathbf{h}_{\text {sex }}(f):=\inf \left\{\mathbf{h}_{\text {top }}(g)\right\},
$$

where the inf is over all symbolic extensions of $f$. (If there is no symbolic extension of $(X, f)$, then $\mathbf{h}_{\mathrm{sex}}(f)=\infty$.) The topological residual entropy of $f$ is $\mathbf{h}_{\text {res }}(f):=\mathbf{h}_{\text {sex }}(f)-\mathbf{h}_{\text {top }}(f)$.

If $\mathbf{h}_{\text {top }}(f)=0$, then $\mathbf{h}_{\text {sex }}(f)=0$; otherwise, the residual and topological entropies are independent, as follows.

THEOREM 6.3 ([14; 27]). If $0<\alpha<\infty$ and $0 \leq \beta \leq \infty$ then there is a homeomorphism $f$ with $h_{\text {top }}(f)=\alpha$ and $h_{\text {res }}(f)=\beta$.

Let $\mu_{f}$ be the space of $f$-invariant Borel probabilities, and let $h$ denote the entropy function on $M_{f}, h(\mu)=h(\mu, f)$. The key to a good entropy theory for symbolic extensions [12] is to study the extensions in terms of $\mu_{f}$ (as begun in [27]).

Definition 6.4. Let $\varphi:(Y, S) \rightarrow(X, f)$ be a symbolic extension of $(X, f)$. The extension entropy function of $\varphi$ is the function

$$
\begin{aligned}
h_{\mathrm{ext}}^{\varphi}: \mu_{f} & \rightarrow[0,+\infty) \\
\mu & \mapsto \sup \left\{h(v, S): v \in \mathcal{M}_{g}, \varphi v=\mu\right\} .
\end{aligned}
$$

For a given $\mu \in \mathcal{M}_{f}$ and a given symbolic extension $\varphi$, the number $h_{\mathrm{ext}}^{\varphi}(\mu)$ measures the information in the symbolic system used to encode the trajectories in the support of $\mu$. Define the symbolic extension entropy function of $f$,

$$
\begin{aligned}
h_{\mathrm{sex}}^{f}: \mu_{f} & \rightarrow[0, \infty) \\
\mu & \mapsto \inf _{\varphi} h_{\mathrm{ext}}^{\varphi}(\mu),
\end{aligned}
$$


where the inf is over all symbolic extensions $\varphi$ of $(X, f)$. (If there is no symbolic extension of $(X, f)$, define $h_{\mathrm{sex}}^{f}:=\infty$.) The function $h_{\mathrm{sex}}^{f}$ is capturing for all $\mu$ in $\mu_{f}$ the lower bound on the information required in any finite encoding of the system (i.e., any symbolic extension of $(X, f)$ ) to describe the trajectories supported by $\mu$. This function is a highly refined quantitative reflection of the emergence of chaos (entropy) in the system $(X, f)$, as it reflects "where" the chaos arises (on the supports of which measures) and also "how" (as the scale of resolution on which the system is examined refines to zero). There is a more thorough elaboration of this intuition in [28]; in any case, the final justification of the claim is the full theory of entropy structure $[12 ; 28]$.

To make this precise we follow the path of $[27 ; 12 ; 28]$ and study an allowed sequence of functions $h_{n}: \mu_{f} \rightarrow[0, \infty)$ which increase to $h$. An allowed sequence determines the entropy structure of $(X, f)$. There are many choices of allowed sequence for $\left(h_{n}\right)$, studied in [28]; here is one concrete and crucial (though not completely general) example, which reflects the intuition of "refining scales". Suppose $X$ admits a refining sequence of finite partitions $\mathscr{P}_{n}$, with diameters of partition elements going to zero uniformly in $n$, and such that the boundary of $P$ has $\mu$ measure zero, for all $\mu$ in $\mu_{f}$, for all $n$, and for all $P$ in $\mathscr{P}_{n}$. (Such partitions exist for example if $X$ is finite dimensional with zerodimensional periodic point set [93] or if $(X, f)$ has an infinite minimal factor [101; 99].) Set $h_{n}(\mu)=h_{n}\left(\mu, f, \mathscr{P}_{n}\right)$. Then $\left(h_{n}\right)$ defines the entropy structure.

In [12], one general construction of the entropy structure is given, and the collection of all the functions $h_{\mathrm{ext}}^{\varphi}$ is given a useful functional analytic characterization in terms of the entropy structure. Together with [29], this reduced many problems involving symbolic extensions to problems of pure functional analysis on a metrizable Choquet simplex. For example, it became possible [12] to show the following

- There is a homeomorphism $f$ with $h_{\text {res }}(f)<\infty$, but with no symbolic extension $(Y, g)$ such that $h_{\text {top }}(g)=h_{\text {top }}(f)+h_{\text {res }}(f)$.

- The function $h_{\mathrm{sex}}^{f}$ is upper semicontinuous and its maximum need not be achieved at any ergodic measure.

- The topological symbolic extension entropy of $f$ is the maximum value achieved by its symbolic extension entropy function.

Another outcome was an inductive characterization of the function $h_{\mathrm{sex}}^{f}$ from the given sequence $h_{n}$. Define the tail sequence $\tau_{n}:=h-h_{n}$, which decreases to zero. For ordinals $\alpha, \beta$, define recursively

- $u_{0} \equiv 0$

- $u_{\alpha+1}=\lim _{k}\left(\widetilde{u_{\alpha}+\tau_{k}}\right)$

- $u_{\beta}=$ the u.s.c. envelope of $\sup \left\{u_{\alpha}: \alpha<\beta\right\}$, if $\beta$ is a limit ordinal. 
With these definitions, there is the following theorem.

THEOREM 6.5 [12; 28]. $u_{\alpha}=u_{\alpha+1} \Longleftrightarrow u_{\alpha}+h^{f}=h_{\mathrm{sex}}^{f}$, and such an $\alpha$ exists among countable ordinals (even if $h_{\mathrm{sex}} \equiv \infty$ ).

The convergence above can be transfinite [12], and this indicates the subtlety of the emergence of complexity on ever smaller scales. However the characterization is also of practical use for constructing examples.

Downarowicz unified the whole theory with an appropriate notion of equivalence. Following [28], declare two nondecreasing sequences of nonnegative functions $\left(h_{n}\right)$ and $\left(h_{n}^{\prime}\right)$ to be uniformly equivalent if for every integer $n$ and $\varepsilon>0$ there exists $N$ such that $h_{N}>h_{n}^{\prime}-\varepsilon$ and $h_{N}^{\prime}>h_{n}-\varepsilon$. Now, let $\left(h_{n}\right)$ be a sequence defining the entropy structure in [12] (given by a complicated general construction from [12]). Let $\left(h_{n}^{\prime}\right)$ be another nondecreasing sequence of nonnegative functions on $\mu_{f}$. Then by definition, $\left(h_{n}^{\prime}\right)$ also defines the entropy structure if and only if it is uniformly equivalent to the reference sequence $\left(h_{n}\right)$. Thus the entropy structure for a system $(X, f)$ is a certain uniform equivalence class of sequences of functions on $M_{f}$. The many approaches to defining entropy lead to many candidate sequences $\left(h_{n}\right)$, and Downarowicz examined them [28]. With few exceptions, the approaches yield sequences in the same uniform equivalence class as the reference sequence (and most of these sequences are considerably more simple to define then the reference sequence). A sequence uniformly equivalent to the reference sequence determines all the same entropy invariants (e.g., the topological entropy, the entropy function on $\mu_{f}, h_{\mathrm{sex}}^{f}$, and the transfinite order of accumulation in Theorem 6.5), by application of the same functional analytic characterizations as apply to derive the invariants from the reference sequence. Because so many sequences lead to the same encompassing collection of entropy invariants, it makes sense to refer to the entire equivalence class of these sequences as the entropy structure of the system.

Viewing the entropy structure as fundamental, one asks which structures can occur. At the level of topological dynamics there is a complete answer, due to Downarowicz and Serafin.

THEOREM 6.6 [29]. The following are equivalent.

(1) $\left(g_{n}\right)$ is a nondecreasing sequence of functions on a metrizable Choquet simplex $C$, beginning with $g_{0} \equiv 0$ and converging to a bounded function $g$, and with $g_{n+1}-g_{n}$ upper semicontinuous for all $n$.

(2) There is a homeomorphism $f$ of a compact metrizable space, with entropy structure given by a sequence $\left(h_{n}\right)$, such that there exists an affine homeomorphism from $\mu_{f}$ to $C$ which takes $\left(h_{n}\right)$ to a sequence uniformly equivalent to $\left(g_{n}\right)$. 
Given $\left(g_{n}\right)$ above, Downarowicz and Serafin actually construct a model $f$ on the Cantor set such that the affine homeomorphism takes $\left(h_{n}\right)$ to $\left(g_{n}\right)$. Moreover, $f$ can be made minimal.

More generally one asks what entropy structures are compatible with what degrees of smoothness.

QUESTION 6.7. Let $X$ be a compact Riemannian manifold and $1 \leq r \leq \infty$. What entropy structures are possible for $C^{r}$ diffeomorphisms on $X$ ?

Precisely, Question 6.7 asks the following: given $\left(g_{n}\right)$ a nondecreasing sequence of nonnegative upper semicontinuous functions on a metrizable Choquet simplex $C$, and converging to a bounded function $g$, does there exist a $C^{r}$ diffeomorphism $f$ on $X$, with entropy structure given by a sequence $\left(h_{n}\right)$, such that there exists an affine homeomorphism from $\mu_{f}$ to $C$ which takes $\left(h_{n}\right)$ to a sequence uniformly equivalent to $\left(g_{n}\right)$ ?

Question 6.7 is more a program for the decades than one problem. We move to particular (still very difficult) problems within this program.

First, we isolate the one good distinguished class in the entropy structure theory: this is the Misiurewicz class of asymptotically h-expansive systems [109]. It turns out that $(X, f)$ is asymptotically $h$-expansive if and only if its entropy structure is given by a sequence $\left(h_{n}\right)$ which converges to $h$ uniformly [28], if and only if it has a principal symbolic extension in the sense of Ledrappier (the factor map preserves the entropy of every invariant measure) $[14 ; 12]$. Buzzi showed a $C^{\infty}$ system $(X, f)$ is asymptotically h-expansive [19].

QUESTION 6.8. Which asymptotically h-expansive entropy structures occur for some $C^{\infty}$ diffeomorphism on some (or a given) compact manifold $X$ ?

Note that the Newhouse conjecture (Conjecture 7.1) implies severe constraints to realization if $X$ is a surface.

At the 1991 Yale conference for Roy Adler, Boyle presented the first examples of systems with finite entropy but with no symbolic extension (these were constructed in response to a 1988 question of Joe Auslander). This provoked a question from A. Katok.

QUESTION 6.9 (KATOK, 1991). Are there smooth finite entropy examples with no symbolic extension?

We have seen that there are no bad $C^{\infty}$ examples. For lesser smoothness, Downarowicz and Newhouse showed that the situation is quite different.

THEOREM 6.10 [30]. A generic area-preserving $C^{1}$ diffeomorphism of a surface is either Anosov or has no symbolic extension. If $1<r<\infty$ and $\operatorname{dim}(M)>$ 1 then there are residual subsets $\mathscr{R}$ of open sets in $\operatorname{Diff}^{r}(M)$ such that $h_{\text {res }}(f)>$ 0 - and hence $f$ has no principal symbolic extension - for every $f \in \Re$. 
The first result implies that a generic area-preserving $C^{1}$ surface diffeomorphism that is not Anosov is not topologically conjugate to any $C^{\infty}$ diffeomorphism; this includes all diffeomorphisms on surfaces other than $\mathbb{T}^{2}$. The difficult proof of [30] merges the detailed entropy theory of symbolic extensions with genericity arguments for persistent homoclinic tangencies. Concrete examples of $C^{r}$ maps $1 \leq r<\infty$ with positive residual entropy, based on old examples of Misiurewicz [107; 108], are given in [13]. The most important open problem currently is the following.

QUESTION 6.11. Suppose $f$ is a $C^{r}$ diffeomorphism of a compact Riemannian manifold, with $1<r<\infty$. Is it possible for $f$ to have infinite residual entropy?

The arguments of [30] led Downarowicz and Newhouse to the following more specific version of this problem.

QUESTION 6.12. Let $M$ be a compact manifold, $f: M \rightarrow M a C^{r}$ map with $r>1$. Is it necessarily true that

$$
h_{\text {sex }}(f) \leq h_{\text {top }}(f)+\frac{\operatorname{dim} M \log \operatorname{Lip}(f)}{r-1} ?
$$

The right-hand side here is effectively an iterated Yomdin-type defect. Yomdin proved that the defect in upper semicontinuity given by local volume growth is $\operatorname{dim} M \log \operatorname{Lip}(f) / r$. In the constructions one tends to carry out in this field, one iterates the procedure that gives this estimate and divides again by $r$ each time. The right-hand side above is the sum of the resulting geometric series. For $r=1$ and $r=\infty$ this right-hand side agrees with the known results: $C^{1}$-maps may not have a symbolic extension at all, and $C^{\infty}$ maps have a principal symbolic extension. The question is related to the sense that maps of intermediate regularity should have symbolic extensions, and the entropies of these should not be too much larger than that of a map and by a margin that is less for maps with higher regularity.

\section{Measures of maximal entropy (presented by Sheldon Newhouse)}

CONJECTURE 7.1 (Newhouse). Let $M$ be a compact surface and $f: M \rightarrow M$ a $C^{\infty}$ diffeomorphism with $h_{\text {top }}(f)>0$. Then there are at most finitely many measures of maximal entropy.

Evidence for this conjecture can be found in many places. Franz Hofbauer essentially proved the analogous fact for piecewise monotone maps of the interval.

There are a countable number of homoclinic closures, and all ergodic measures of sufficiently high entropy are supported on these. 
The product of an Anosov diffeomorphism of $\mathbb{T}^{2}$ with the identity on the circle shows that in higher dimension such a claim can only hold with some additional hypotheses.

\section{Properties of the measure-theoretic entropy of Sinai-Ruelle-Bowen measures of hyperbolic attractors (contributed by Miaohua Jiang)}

Let Diff ${ }^{1+\alpha}(M)$ be the collection of all $C^{1+\alpha}$-diffeomorphisms on a compact smooth Riemannian manifold $M$. Assume that a map $f_{0} \in \operatorname{Diff}^{1+\alpha}(M)$ is transitive and has a hyperbolic attractor $\Lambda$ as its nonwandering set. By structural stability, any $g \in \operatorname{Diff}^{1+\alpha}(M)$ in a sufficiently small $C^{1}$-neighborhood of $f_{0}$ is topologically conjugate to $f_{0}$ on the attractor and its nonwandering set is also a hyperbolic attractor. We denote this neighborhood of $f_{0}$ by $U_{\varepsilon}^{C^{1}}\left(f_{0}\right)$. Let $U\left(f_{0}\right)$ be the collection of those diffeomorphisms in Diff ${ }^{1+\alpha}(M)$ that can be connected with $f_{0}$ by a finite chain of such neighborhoods, i.e.,

$$
\begin{aligned}
U\left(f_{0}\right)= & \left\{g \mid \exists n \in \mathbb{N} \forall i=1,2, \ldots, n \exists f_{i} \in \operatorname{Diff}^{1+\alpha}(M), \varepsilon_{i}>0:\right. \\
& \left.g \in U_{\varepsilon_{n}}^{C^{1}}\left(f_{n}\right) \text { and } U_{\varepsilon_{i-1}}^{C^{1}}\left(f_{i-1}\right) \cap U_{\varepsilon_{i}}^{C^{1}}\left(f_{i}\right) \neq \varnothing \text { for } i=1,2, \ldots, n\right\} .
\end{aligned}
$$

The set $U\left(f_{0}\right)$ is an open set of $\operatorname{Diff}^{1+\alpha}(M)$. Any map $f$ in $U\left(f_{0}\right)$ possesses a hyperbolic attractor and there exists an SRB measure $\mu_{f}$ for $f$. Any two maps in $U\left(f_{0}\right)$ are conjugate by a Hölder continuous map that is not necessarily close to the identity. Each map in $U\left(f_{0}\right)$ also has the same topological entropy. However, the measure-theoretic entropy $h_{\mu_{f}}(f)$ of $f \in U\left(f_{0}\right)$ with respect to its SRB measure $\mu_{f}$ can vary. It was shown by David Ruelle that the dependence of $\mu_{f}$ on the map $f$ is differentiable when the maps are $C^{4}$.

QUESTION 8.1. Is $\inf _{f \in U\left(f_{0}\right)} h_{\mu_{f}}(f)=0$ ? (Added in proof: Miaohua Jiang, Huyi Hu and Yunping Jiang have answered the question in the affirmative.)

\section{QUESTION 8.2. Does this functional have a local minimum?}

For expanding maps on the circle, the infimum being zero was confirmed by Mark Pollicott. The problems were raised during conversations between Miaohua Jiang and Dmitry Dolgopyat.

\section{Sinai-Ruelle-Bowen measures and natural measures (presented by Michał Misiurewicz)}

DEFINITION 9.1. Let $X$ be a compact metric space, $f: X \rightarrow X$ a continuous map, $\mu$ the space of all probability measures on $X$ and $f_{*}: \mathcal{M} \rightarrow \mathcal{M},\left(f_{*}(\mu)\right) \varphi:=$ $\mu(\varphi \circ f)$, where $\mu(\varphi):=\int_{X} \varphi d \mu$ for $\varphi: X \rightarrow \mathbb{R}$. Given a "reference" measure 
$m$ on $X$ for which $f_{*}(m) \ll m$ and $A_{n}(\mu):=\sum_{k=0}^{n-1} f_{*}^{k}(\mu) / n$, a Sinai-RuelleBowen measure for $f$ is a measure $m_{f}$ such that there is an open $U \subset X$ with $m(U)>0$ such that $\lim _{n \rightarrow \infty} A_{n}\left(\delta_{x}\right)=m_{f}$ for $m$-a.e. $x \in U[135 ; 136]$. A natural measure is a measure $m_{f}$ for which there is an open set $U$ with $m(U)>0$ such that $\lim _{n \rightarrow \infty} A_{n}(\mu)=m_{f}$ for every $\mu \in \mathcal{M}$ with $\mu(U)=1$ and $\mu \ll m$.

THEOREM 9.2. A Sinai-Ruelle-Bowen measure is natural.

Proof. Integrate $A_{n}\left(\delta_{x}\right)$ (which tends to $m_{f}$ as $n \rightarrow \infty$ ) over $x$ with respect to $m$.

The converse does not hold.

THEOREM $9.3[110 ; 111 ; 71]$. If $g$ is an expanding algebraic endomorphism or an algebraic Anosov automorphism of a torus $\mathbb{\mathbb { V }}^{d}$ then there exists $f: \mathbb{\mathbb { T }}^{d} \rightarrow \mathbb{\mathbb { T }}^{d}$ that is topologically conjugate to $g$ and such that

(1) $f_{*}(m) \ll m$ for $m=$ Lebesgue measure,

(2) $\lim _{n \rightarrow \infty} A_{n}(m)=m_{f}$,

(3) $m_{f}$ has maximal entropy,

(4) $\left\{A_{n}\left(\delta_{x}\right) \mid n \in \mathbb{N}\right\}$ is dense in the space of $f$-invariant Borel probability measures for $m$-a.e. $x \in \mathbb{T}^{d}$.

This particular situation is impossible for smooth (even $C^{1}$ ) $f$, which motivates

QUESTION 9.4. With Lebesgue measure as the reference measure, are there smooth dynamical systems for which the ergodic natural measure is not a SinaiRuelle-Bowen measure?

There is an example of piecewise continuous, piecewise smooth interval map for which the natural measure is the average of two delta-measures at fixed points and an SRB measure does not exist [10, page 391].

One could say that in these examples the conjugacy sends Lebesgue measure to one that is completely unrelated to Lebesgue measure.

\section{Billiards (Domokos Szasz)}

Consider a dispersing billiard on the two-dimensional torus with a finite horizon (i.e., assume that the length of orbit segments between impacts with scatterers is bounded). The Lorentz process is the $\mathbb{Z}^{2}$-cover of this billiard. (In other words, it is a billiard on $\mathbb{R}^{2}$ with periodically arranged convex scatterers.) The phase space of the billiard can, of course, be embedded isomorphically into the phase space of the Lorentz process, into its cell 0, say. Assume that the initial phase point of the Lorentz process is selected in cell 0 according to the Liouville measure. 
It is known that, for the billiard dynamics, correlations of Hölder functions decay exponentially fast (cf. [136]). As a consequence, for Hölder functions the central limit theorem holds, implying that for the corresponding Lorentz process the typical displacement of orbits increases as the square root of the number of collisions. However, for periodic trajectories of the billiard the displacement is either bounded or is ballistic (i.e., it grows linearly with the number of collisions). According to a construction of [16] (for more details and further references see [132]) there do exist ballistic orbits.

QUESTION 10.1. How large is the set of ballistic orbits? Could one give a lower bound for its Hausdorff dimension?

This is a geometric question because it is not a matter of studying typical behavior. When one aims at constructing ballistic orbits different from those arising from periodic ones the problem is that there are "shadows" of the scatterers, which makes this situation different from geodesic flows in negative curvature because it introduces an analog of positive curvature, and there is no good geometric picture here.

\section{Stable ergodicity (with Keith Burns)}

DEFINITION 11.1. An embedding $f$ is said to be partially hyperbolic on $\Lambda$ if there exists a Riemannian metric for which there are continuous positive functions $\lambda_{i}, \mu_{i}, i=1,2,3$ on $M$ such that

$$
0<\lambda_{1} \leq \mu_{1}<\lambda_{2} \leq \mu_{2}<\lambda_{3} \leq \mu_{3} \text { with } \mu_{1}<1<\lambda_{3}
$$

and an invariant splitting

$$
T_{x} M=E^{s}(x) \oplus E^{c}(x) \oplus E^{u}(x), \quad d_{x} f E^{\tau}(x)=E^{\tau}(f(x)), \tau=s, c, u
$$

into pairwise orthogonal subspaces $E^{s}(x), E^{c}(x)$ and $E^{u}(x)$ such that

$$
\begin{aligned}
& \left.\lambda_{1} \leq \| d_{x} f \uparrow E^{s}(x)\right\rfloor\|\leq\| d_{x} f \uparrow E^{s}(x) \| \leq \mu_{1}, \\
& \lambda_{2} \leq\left\|d_{x} f \uparrow E^{c}(x)\right\|\|\leq\| d_{x} f \uparrow E^{c}(x) \| \leq \mu_{2}, \\
& \lambda_{3} \leq\left\|\left\lfloor d_{x} f \uparrow E^{u}(x)\right\rfloor\right\| \leq\left\|d_{x} f \uparrow E^{u}(x)\right\| \leq \mu_{3},
\end{aligned}
$$

where $\|\lfloor A\rfloor\|:=\min \{\|A v\| \mid\|v\|=1\}$. In this case we set $E^{c s}:=E^{c} \oplus E^{s}$ and $E^{c u}:=E^{c} \oplus E^{u}$.

REMARK. Each subbundle $E^{\tau}$ for $\tau=u, s, c, c u, c s$ is Hölder continuous.

Denote the set of $C^{2}$ partially hyperbolic diffeomorphisms of a compact manifold $M$ by $\operatorname{PHD}^{2}(M)$ and the set of volume-preserving such by $\operatorname{PHD}_{\mathrm{vol}}^{2}(M)$.

Conjecture 11.2 (Pugh-Shub). The set of diffeomorphisms that are volume-ergodic contains a $C^{2}$-dense and $C^{1}$-open subset of $P H D_{\mathrm{vol}}^{2}(M)$. 
Since the usual method available for establishing ergodicity from hyperbolicity is the Hopf argument, it is natural to consider $u s$-paths, that is, paths obtained by concatenating finitely many segments each of which lies entirely in a stable or an unstable leaf. The property of being joined by a $u s$-path is obviously an equivalence relation on points of $M$. If there is just one equivalence class, in other words if any two points are joined by a $u s$-path, we say that the diffeomorphism has the accessibility property. One also wants to consider this property modulo sets of measure 0, which leads to the essential accessibility property, which says that a measurable set which is a union of equivalence classes must have zero or full measure.

This suggests approaching the above conjecture via the two following ones: CONJECTURE 11.3. $P H D_{\mathrm{vol}}^{2}(M)$ and $P H D^{2}(M)$ contain subsets consisting of diffeomorphisms with the accessibility property that are both $C^{2}$-dense and $C^{1}$-open.

Conjecture 11.4. Essential accessibility implies ergodicity in $P H D_{\mathrm{vol}}^{2}(M)$.

Pertinent known results are:

THeOREM 11.5 [26]. Conjecture 11.3 is true if $C^{2}$ dense is weakened to $C^{1}$ dense.

THEOREM 11.6 [114; 65; 17]. Conjecture 11.3 is true for diffeomorphisms with 1-dimensional center.

Removing the assumption of 1-dimensional center bundle will require substantially new ideas.

Results towards Conjecture 11.4 are the classical ones by Hopf [66], Anosov and Anosov-Sinai $[1 ; 3]$ as well as those by Grayson, Pugh and Shub [52; 123], Pugh and Shub [124; 125] and the most refined version due to Burns and Wilkinson [18].

Definition 11.7. We say that $f$ is center-bunched if $\mu_{1}<\lambda_{2} / \mu_{2}$ and $\lambda_{3}^{-1}<$ $\lambda_{2} / \mu_{2}$.

This holds automatically whenever the center bundle is 1-dimensional.

THEOREM 11.8 [18]. An (essentially) accessible, center-bunched, partially hyperbolic diffeomorphism is ergodic (and, in fact, has the K-property).

With this in mind one can rephrase Conjecture 11.4 as follows:

QUESTION 11.9. Can one dispense with the center-bunching hypothesis in Theorem 11.8?

This would require a substantially new insight. The present techniques crucially require center bunching, even though it has been weakened significantly from its earliest formulations. 
Maybe a different approach is needed:

QUESTION 11.10. Can one show that accessibility implies ergodicity of the stable and unstable foliations in the sense that sets saturated by stable leaves and sets saturated by unstable leaves must have either zero or full measure?

It is not known whether a diffeomorphism that satisfies the hypotheses of Theorem 11.8 must be Bernoulli.

QUESTION 11.11. Are systems as in Theorem 11.8 Bernoulli?

The answer is expected to be negative, but the known examples of K-systems that are not Bernoulli are not of this type. It may be possible that a study of early smooth examples by Katok may be instructive. They are not partially hyperbolic but might be sufficiently "soft" to be useful here.

\section{Mixing in Anosov flows (Michael Field)}

Let $\Lambda$ be a basic set for the Axiom A flow $\Phi$ and $\mathscr{P}$ denote the periodic spectrum of $\Phi \uparrow \Lambda$ (set of prime periods of periodic orbits). Bowen showed that $\mathscr{P}$ is an invariant of mixing. The analyticity and extension properties of the $\zeta$-function $\zeta_{\Phi}$ of $\Phi$ are (obviously) determined by $\mathscr{P}$ (for the definition of $\zeta_{\Phi}$ we assume the measure of maximal entropy on $\Lambda$ ). In view of the close relation between exponential mixing of $\Phi$ and extension properties of $\zeta_{\Phi}$ [122], we ask

QUESTION 12.1. Is the periodic spectrum an invariant of exponential mixing?

(For conditions on $\mathscr{P}$ related to rapid mixing, see [41, Theorem 1.7].)

Let $x$ be a homoclinic point for the periodic orbit $\Gamma$. In [41] a definition is given of 'good asymptotics' for the pair $(\Gamma, x)$. Without going into detail, the definition involves precise asymptotic estimates for a sequence of periodic orbits which converge to the $\Phi$-orbit of $x$. Typically, good asymptotics is an open condition in the $C^{2}$-topology. If there exists $(\Gamma, x)$ with good asymptotics then $\Phi$ is (rapidly) mixing [41].

DEFINITION 12.2. We say $\Phi$ has very good asymptotics if every pair $(\Gamma, x)$ has good asymptotics in the sense of [41].

This is a generic condition on Axiom A flows.

QUESTION 12.3. Does very good asymptotics imply exponential mixing?

A weaker (but perhaps more tractable) version of this question is

QUESTION 12.4. Does very good asymptotics imply analytic extension of the $\zeta$-function? 
A flow $\Phi$ is $C^{r}$-stably mixing if there exists a $C^{r}$-open neighborhood of $\Phi$ consisting of mixing flows. It was shown in [41] that if $r \geq 2$ then a $C^{r}$-Axiom A flow can be $C^{r}$-approximated by a $C^{2}$-stably mixing Axiom A flow (in fact, by a $C^{2}$-stably rapid mixing flow). If the flow is Anosov or an attractor one may approximate by a $C^{1}$-stably mixing flow.

QUESTION 12.5. If the dimension of the basic set is at least two, can one always approximate by a $C^{1}$-stably mixing flow?

(This is really a question about the local geometry of the basic set. For example, it suffices to know that $W^{u u}(x) \cap \Lambda$ is locally path-connected for all $x \in \Lambda$. This condition is automatically satisfied for attractors.)

For results and background related to the following question see [40].

Problem 12.6. Suppose the dimension of the basic set is one (suspension of a subshift of finite type). Show that if $\Phi$ is $C^{1}$-stably mixing, then $\Phi$ cannot be $C^{r}, r>1$.

Of course, it is interesting here to find examples where $C^{1+\alpha}$-stable mixing of $\Phi$ implies that $\Phi$ cannot be more regular than $C^{1+\alpha}$.

Although the results in [41] show that every Anosov flow can be approximated by a $C^{1}$-stably mixing Anosov flow, there remains the

Conjecture 12.7 (Plante [121]). For transitive Anosov flows, mixing is equivalent to stable mixing.

As Plante showed, the conjecture amounts to showing that if the strong foliations are integrable then they cannot have dense leaves.

\section{The structure of hyperbolic sets (contributed by Todd Fisher)}

As stated in Section 16 below, there are a number of fundamental questions about the structure of Anosov diffeomorphisms. It is then not surprising that there are a number of problems concerning the structure of general hyperbolic sets.

A question posed by Bonatti concerns the topology of hyperbolic attractors.

On surfaces a hyperbolic attractor can be either the entire manifold (Anosov case) or a 1-dimensional lamination ("Plykin attractors").

On 3-dimensional manifolds there are many kinds of hyperbolic attractors: Let $A$ be a hyperbolic attractor of a diffeomorphism $f$ on a compact 3-manifold $M$. The following cases are known to exist.

(1) If the unstable manifold of the points $x \in A$ are bidimensional, then $A$ is either the torus $T^{3}$ (Anosov case), or a bidimensional lamination. 
(2) If the unstable manifolds of the points $x \in A$ are 1-dimensional, then the attractor can be

(i) a 1-dimensional lamination which is transversely Cantor ("Williams attractors") or

(ii) an invariant topological 2-torus $\mathbb{T}^{2}$, and the restriction of $f$ to this torus is conjugate to an Anosov diffeomorphism (however, the torus $\mathbb{T}^{2}$ can be fractal with Hausdorff dimension strictly bigger than 2).

QUESTION 13.1. Is there some other possibility? For example, is it possible to get an attractor such that the transversal structure of the unstable lamination is a Sierpinski carpet?

THEOREM 13.2 [42]. If $M$ is a compact surface and $\Lambda$ is a nontrivial mixing hyperbolic attractor for a diffeomorphism $f$ of $M$, and $\Lambda$ is hyperbolic for a diffeomorphism $g$ of $M$, then $\Lambda$ is either a nontrivial mixing hyperbolic attractor or a nontrivial mixing hyperbolic repeller for $g$.

It is also shown in [42] by counterexample that this does not hold in higher dimensions for general attractors. However, if we add some assumptions on the attractor or weaken the conclusion we have the following problems.

QUESTION 13.3. Suppose $M$ is a compact smooth boundaryless manifold of dimension $n$ and $\Lambda$ is a mixing hyperbolic attractor for $f$ with $\operatorname{dim}\left(\mathbb{E}^{u}\right)=n-1$ and hyperbolic for a diffeomorphism $g$. Does this imply that $\Lambda$ is a mixing hyperbolic attractor or repeller for $g$ ?

QUESTION 13.4. Suppose $\Lambda$ is a locally maximal hyperbolic set for a diffeomorphism $f$ and hyperbolic for a diffeomorphism $g$. Does this imply that $\Lambda$ is locally maximal for $g$ ? or that $\Lambda$ is contained in a locally maximal hyperbolic set for $g$ ?

Related to Question 13.4 we note that in [43] it is shown that on any manifold, of dimension greater than one, there is an open set of diffeomorphisms containing a hyperbolic set that is not contained in a locally maximal one. Furthermore, it is shown if the dimension of the manifold is at least four that there is an open set of diffeomorphisms containing a transitive hyperbolic set that is not contained in a locally maximal one.

QUESTION 13.5. Suppose $M$ is a compact surface and $\Lambda \subset M$ is a transitive hyperolic set for a diffemorphism $f$ of $M$. If $\Lambda$ is transitive, then is $\Lambda$ contained in a locally maximal hyperbolic set?

Inspired by Hilbert's famous address in 1900, Arnold requested various mathematicians to provide great problems for the twenty-first century. Smale gave his list in [130]. Smale's Problem 12 deals with the centralizer of a "typical" 
diffeomorphism. For $f \in \operatorname{Diff}^{r}(M)$ (the set of $C^{r}$ diffeomorphisms from $M$ to $M$ ) the centralizer of $f$ is

$$
C(f)=\left\{g \in \operatorname{Diff}^{r}(M) \mid f g=g f\right\} .
$$

Let $r \geq 1, M$ be a smooth, connected, compact, boundaryless manifold, and

$$
T=\left\{f \in \operatorname{Diff}^{r}(M) \mid C(f) \text { is trivial }\right\} .
$$

Smale asks whether $T$ is dense in $\operatorname{Diff}^{r}(M)$. Smale also asks if there is a subset of $T$ that is open and dense in $\operatorname{Diff}^{r}(M)$. Smale states: "I find this problem interesting in that it gives some focus in the dark realm beyond hyperbolicity where even the problems are hard to pose clearly." [130]

Even though Smale states that the problem of studying the centralizer gives focus on nonhyperbolic behavior, unfortunately even the hyperbolic case, in general, remains open. However, a number of people have partial results to Smale's question for Axiom A diffeomorphisms.

Palis and Yoccoz [115] have shown that there is an open and dense set of $C^{\infty}$ Axiom A diffeomorphisms with the strong transversality property and containing a periodic sink that have a trivial centralizer. Togawa [133] has shown that on any manifold there is a $C^{1}$ residual set among $C^{1}$ Axiom A diffeomorphisms with a trivial centralizer.

QUESTION 13.6. For any manifold and any $r \geq 1$ is there an open and dense set $U$ contained in the set of $C^{r}$ Axiom A diffeomorphisms such that any $f \in U$ has a trivial centralizer.

\section{The dynamics of geodesic flows (presented by Gerhard Knieper)}

CONJECTURE 14.1. For any compact manifold the geodesic flow of a generic Riemannian metric has positive topological entropy.

This holds for surfaces. Specifically, for tori this is achieved using methods of Hedlund, Birkhoff and others to construct a horseshoe, and for higher genus this is a consequence of the exponential growth forced by entropy. Consequently only the sphere requires substantial work. For the sphere Contreras and Paternain [21] showed this in the $C^{2}$-topology (for metrics) using dominated splitting and Knieper and Weiss [92] proved this in the $C^{\infty}$ topology using global Poincaré sections (pushed from the well-known case of positive curvature using work of Hofer and Wysocki in symplectic topology) and the theory of prime ends as applied by Mather. A consequence (via a theorem of Katok) is that generically there is a horseshoe and hence exponential growth of closed geodesics.

QUESTION 14.2. Can one make similar statements for Liouville entropy? 
QUESTION 14.3. Is there a metric of positive curvature whose geodesic flow has positive Liouville entropy?

The underlying question is whether there is a mechanism for the generation of hyperbolicity on a large set from positive curvature.

If a manifold of nonpositive curvature has rank 1 (i.e., every geodesic is hyperbolic), then the unit tangent bundle splits into two sets that are invariant under the geodesic flow, the regular set, which is open and dense, and the singular set.

QUESTION 14.4. Does the singular set have zero Liouville measure?

An affirmative answer would imply ergodicity of the geodesic flow. For analytic metrics on surfaces the singular set is a finite union of closed geodesics. In higher dimensions there are examples where the singular set carries positive topological entropy.

Irreducible nonpositively curved manifolds of higher rank are locally symmetric spaces by the rank rigidity theorem. In this case closed geodesics are equidistributed. For $\varepsilon>0$ let $P_{\varepsilon}(M)$ be a maximal set of $\varepsilon$-separated closed geodesics and $P_{\varepsilon}(t):=\left\{c \in P_{\varepsilon}(M) \mid \ell(c) \leq t\right\}$. By a result of Spatzier there is an $\varepsilon>0$ such that $\lim _{t \rightarrow \infty}(1 / t) \log \operatorname{card} P_{\varepsilon}(t)=h_{\text {top }}\left(\varphi^{t}\right)$. This implies that closed geodesics are equidistributed with respect to the measure of maximal entropy.

QUESTION 14.5. Can one replace "E-separated" by "nonhomotopic"?

This is likely but unknown.

\section{Averaging (Yuri Kifer)}

The basic idea in averaging is to start from an "ideal" (unperturbed) system

$$
\begin{array}{ll}
\frac{d X(t)}{d t}=0, & X(0)=0, \\
\frac{d Y(t)}{d t}=b(x, Y(t)), & Y(0)=y,
\end{array}
$$

which gives rise to the flow $\varphi_{0}^{t}: \mathbb{R}^{d} \times M \rightarrow \mathbb{R}^{d} \times M,(x, y) \mapsto\left(x, F_{x}^{t}(y)\right)$. Integrable Hamiltonian systems are of this type. One then perturbs the system by adding a slow motion in the first coordinate:

$$
\begin{array}{ll}
\frac{d X^{\varepsilon}(t)}{d t}=\varepsilon B\left(X^{\varepsilon}(t), Y^{\varepsilon}(t)\right), & X^{\varepsilon}(0)=x, \\
\frac{d Y^{\varepsilon}(t)}{d t}=b\left(X^{\varepsilon}(t), Y^{\varepsilon}(t)\right), & Y^{\varepsilon}(0)=y .
\end{array}
$$


We write $X^{\varepsilon}=X_{x, y}^{\varepsilon}$ and $Y^{\varepsilon}=Y_{x, y}^{\varepsilon}$. This results in a flow

$$
\varphi_{\varepsilon}^{t}(x, y)=\left(X_{x, y}^{\varepsilon}(t), Y_{x, y}^{\varepsilon}(t)\right),
$$

with $X$ representing the slow motion. The question is whether on a time scale of $t / \varepsilon$ the slow motion can be approximated by solving the averaged equation where $B$ is replaced by $\bar{B}$ which is obtained from the former by averaging it along the fast motion (see [89] and [90]).

$$
\frac{\bar{X} \varepsilon(t)}{d t}=\varepsilon \bar{B}\left(X^{\varepsilon}(t)\right)
$$

In discrete time the "ideal" unperturbed system is of the form

$$
\varphi_{0}(x, y)=\left(x, F_{x}(y)\right),
$$

with $x \in \mathbb{R}^{d}, F_{x}: M \rightarrow M$. The perturbed system is

$$
\varphi_{\varepsilon}(x, y)=\left(x+\varepsilon \Psi(x, y), F_{x}(y)\right),
$$

and we can bring this into a form analogous to the one for continuous time by writing it as difference equations:

$$
\begin{aligned}
& X^{\varepsilon}(n+1)=X^{\varepsilon}(n)+\varepsilon \Psi\left(X^{\varepsilon}(n), Y^{\varepsilon}(n)\right), \\
& Y^{\varepsilon}(n+1)=F_{X^{\varepsilon}(n)}\left(Y^{\varepsilon}(n)\right) .
\end{aligned}
$$

It is natural to rescale time to $t / \varepsilon$, and a basic averaging result (Artstein and Vigodner [4]) is that (in a sense of differential inclusion) any limit point of

$$
\left\{X^{\varepsilon}(t / \varepsilon) \mid \varepsilon>0, t \in[0, T]\right\}
$$

is a solution of

$$
\frac{d Z^{0}(t)}{d t}=B_{\mu_{z}}\left(Z^{0}(t)\right)
$$

with $Z^{0}(0)=x$, where $\mu_{z}$ is $F_{z}$-invariant and $B_{\mu}(x):=\int B(x, y) d \mu(y)$. Heuristically one instead uses the following averaging principle. Suppose the $\operatorname{limit} \bar{B}(x):=\lim _{t \rightarrow \infty} \int_{0}^{t} B\left(x, F_{x}^{s}(y)\right) d s / t$ exists for "most" $(x, y)$ and "almost" does not depend on $y$. Then try to approximate $X^{\varepsilon}(t)$ in some sense over time intervals of order $1 / \varepsilon$ by the averaged motion $\bar{X}$ given by (15-1) with $\bar{X}^{\varepsilon}(0)=x$. (This goes back to Clairaut, Lagrange, Laplace, Fatou, KrylovBogolyubov, Anosov, Arnold, Neishtadt, Kasuga and others, and there are also stochastic versions.) One then would like to know whether

$$
\lim _{\varepsilon \rightarrow 0} \sup _{0 \leq t \leq T / \varepsilon}\left|X^{\varepsilon}(t)-\bar{X}^{\varepsilon}(t)\right|=0,
$$

and in which sense this happens. Next one can inquire about the error

$$
X^{\varepsilon}(t)-\bar{X}^{\varepsilon}(t)
$$


New results assume that the fast motion is chaotic, typically the second factor is assumed to be hyperbolic. One can then hope for approximation in measure, and there are theorems to that effect in 3 main cases: The fast motion is independent of the slow one (this is the easy case, and one gets a.e.-convergence), when the fast motion preserves a smooth measure that is ergodic for a.e. $x$ (this is due to Anosov and covers the Hamiltonian situation), and much more recently, when the fast motion is an Axiom-A flow (depending $C^{2}$ on $x$ as a parameter) in a neighborhood of an attractor endowed with Sinai-Ruelle-Bowen measure.

One may in the latter case ask whether there is a.e.-convergence rather than in measure. For instance, this is not true in general in the case of perturbations of integrable Hamiltonian systems. In the latter case we may have no convergence for any fixed initial condition from a large open set (see Neishtadt's example in [90]). This is related to the question of whether there are resonances and whether these affect convergence and how. To make this concrete, consider the discrete-time system

$$
\begin{aligned}
& X^{\varepsilon}(n+1)=X^{\varepsilon}(n)+\varepsilon \sin 2 \pi Y^{\varepsilon}(n), \\
& Y^{\varepsilon}(n+1)=2 Y^{\varepsilon}(n)+X^{\varepsilon}(n)(\bmod 1) .
\end{aligned}
$$

QUESTION 15.1. Is it the case that

$$
\lim _{\varepsilon \rightarrow 0} \sup _{0 \leq n \leq T / \varepsilon}\left|X^{\varepsilon}(n)-X^{\varepsilon}(0)\right|=0
$$

for Lebesgue-almost every $(x, y)$ ? (Added in proof: Kifer and Bakhtin now seem to have a negative answer to this.)

It is known by a large-deviations argument (see [90]) that given $\delta>0$ the measure of the set of $(x, y)$ for which $\sup _{0 \leq n \leq T / \varepsilon}\left|X^{\varepsilon}(n)-X^{\varepsilon}(0)\right|>\delta$ is at most $e^{-C / \varepsilon}$ for some $C>0$.

When one considers the rescaled averaged motion $\bar{Z}(t)=\bar{X}^{\varepsilon}(t / \varepsilon)$ (averaged with respect to Sinai-Ruelle-Bowen measure) an adiabatic invariant is an invariant function, i.e., a function $H$ such that $H(\bar{Z}(t))=H(\bar{Z}(0))$.

CONJECTURE 15.2. On $(M, v o l), H\left(X^{\varepsilon}\left(t / \varepsilon^{2}\right)\right)$ converges weakly to a diffusion, assuming that the fast motion is hyperbolic.

It may be simpler to start with expanding fast motions.

\section{Classifying Anosov diffeomorphisms and actions (presented by Anatole Katok and Ralf Spatzier)}

The classical question on which these questions are based is whether one can classify all Anosov diffeomorphisms. This has been done up to topological 
conjugacy on tori and nilmanifolds and for codimension-1 Anosov diffeomorphisms $[48 ; 102 ; 113]$. The central ingredient is the fundamental observation by Franks that if an Anosov diffeomorphism on a torus acts on the fundamental group in the same way as a hyperbolic automorphism then there is a conjugacy. Manning proved that any Anosov map of the torus is indeed of this type and extended the result to nilmanifolds.

QUESTION 16.1. Is every Anosov diffeomorphism of a compact manifold $M$ topologically conjugate to a finite factor of an automorphism of a nilmanifold $N / \Gamma$ ?

If there are indeed other examples, then there is currently a lack of imagination regarding the possibilities for Anosov diffeomorphisms. In the framework of the proofs mentioned above the central assumption is that the universal cover is $\mathbb{R}^{n}$ and the map is globally a product, but there is no a priori reason that this should be so. Indeed, for Anosov flows the situation is quite different, and there are many unconventional examples of these, beginning with one due to Franks and Williams that is not transitive [49].

In higher rank there are plausible mechanisms to rule out topologically exotic discrete Anosov actions.

Perturbation of periodic points shows that one cannot expect better than topological classification of Anosov diffeomorphisms, and an example by Farrell and Jones suggest a different reason: There is an Anosov diffeomorphism on an exotic torus.

One may ask about characterizations of algebraic Anosov actions up to $C^{\infty}$ conjugacy.

Theorem 16.2 [8]. An Anosov diffeomorphism with $C^{\infty}$ Anosov splitting that preserves an affine connection (e.g., is symplectic) is $C^{\infty}$ conjugate to an algebraic one.

CONJECTURE 16.3. Instead of preservation of an affine connection this can be done assuming preservation of some sensible higher-order geometric structure, e.g., a Gromov-rigid structure.

QUESTION 16.4. Does preservation of an affine connection alone suffice?

QUESTION 16.5. Does smooth splitting alone suffice?

This might be possible. A natural approach would be to construct invariant structures on the stable and unstable foliations and glue these together to a global invariant structure. The problem is that in some of the standard nilpotent examples the natural structure is not of the type one gets this way.

A different and more recent result is the following: 
THEOREM 16.6 [76; 32]. A uniformly quasiconformal Anosov diffeomorphism is $C^{\infty}$ conjugate to an algebraic one.

CONJECTURE 16.7 (KATOK). An Anosov diffeomorphism whose measure of maximal entropy is smooth is smoothly conjugate to an algebraic one.

Anosov flows are much more flexible, there are many examples that make a classification seem unlikely $[49 ; 59]$. There are some characterizations of algebraic flows. These provide some results similar to the above, as well as analogous problems.

The situation is rather different for algebraic actions so long as they are irreducible (e.g., not products of Ansov diffeomorphisms). These actions are usually hard to perturb. Katok and Spatzier showed that such actions with semisimple linear part are rigid [85], and Damjanovic and Katok pushed this to partially hyperbolic actions on tori [23].

Conjecture 16.8. Any $C^{\infty}$ Anosov $\mathbb{R}^{k}$ - (or $\mathbb{Z}^{k}$-) action for $k \geq 2$ on a compact manifold without rank 1 factors is algebraic.

No counterexamples are known even with lower regularity than $C^{\infty}$.

We next quote a pertinent result by Federico Rodríguez Hertz:

THEOREM 16.9 [64]. A $\mathbb{Z}^{2}$ action on $\mathbb{T}^{3}$ with at least one Anosov element and whose induced action on homology has only real eigenvalues (one less than 1 and 2 bigger than 1) is $C^{\infty}$ conjugate to an algebraic one.

In fact, more generally, let $\Gamma$ be a subgroup of $\operatorname{GL}(N, \mathbb{Z})$, the group of $N \times N$ matrices with integer entries and determinant \pm 1 , and say that the standard action of $\Gamma$ on $\mathbb{T}^{N}$ is globally rigid if any Anosov action of $\Gamma$ on $\mathbb{T}^{N}$ which induces the standard action in homology is smoothly conjugate to it.

THEOREM 16.10 [64]. Let $A \in G L(N, \mathbb{Z})$, be a matrix whose characteristic polynomial is irreducible over $\mathbb{Z}$. Assume also that the centralizer $Z(A)$ of $A$ in $G L(N, \mathbb{Z})$ has rank at least 2 . Then the associated action of any finite index subgroup of $Z(A)$ on $\mathbb{T}^{N}$ is globally rigid.

The assumption on the rank of the centralizer is hardly restrictive. Due to the Dirichlet unit theorem, in the above case, $Z(A)$ is a finite extension of $\mathbb{Z}^{r+c-1}$ where $r$ is the number of real eigenvalues and $c$ is the number of pairs of complex eigenvalues. So, $r+2 c=N$, and $Z(A)$ has rank 1 only if $N=2$ or if $N=3$ and $A$ has a complex eigenvalue or if $N=4$ and $A$ has only complex eigenvalues.

In [64] Hertz also states:

Question 16.11. Consider $N \geq 3, A \in G L(N, \mathbb{Z})$ such that $Z(A)$ is "big enough". Under which assumptions is the standard action of every finite-index subgroup $\Gamma$ on $\mathbb{T}^{N}$ globally rigid? 
Another rigidity result is due to Kalinin and Spatzier:

THEOREM 16.12 [77]. If $M$ is a compact manifold with a Cartan $\mathbb{R}^{k}$-action such that $k \geq 3$, there is a dense set of Anosov elements and every 1-parameter subgroup is topologically transitive (hence there are no rank-1 factors) then this action is $C^{\infty}$ conjugate to an algebraic one, indeed a homogeneous one (i.e., the left action of $\mathbb{R}^{k}$ embedded in a group $G$ on $G / \Gamma$ for a cocompact discrete subgroup $\Gamma$ ).

(Homogeneous Anosov actions of this type are not classified because it is not known how to classify suspensions of Anosov $\mathbb{Z}^{k}$-actions on nilmanifolds.)

Question 16.13. Does the Kalinin-Spatzier result hold for $k=2$ ?

QUESTION 16.14. Does the Kalinin-Spatzier result hold assuming only the existence of an Anosov element?

QUESTION 16.15. Does the Kalinin-Spatzier result hold without the transitivity assumption (and maybe even without excluding rank-1 factors)?

\section{Invariant measures for hyperbolic actions of higher-rank abelian groups (Anatole Katok)}

The basic example is Furstenberg's " $\times 2 \times 3$ "-example of the $\mathbb{N}^{2}$-action on $S^{1}$ generated by $E_{i}: x \mapsto i x(\bmod 1)$ for $i=1,2$. For a single of these transformations there are plenty of invariant measures, but for $\left\{E_{i} \mid i \in \mathbb{N}\right\}$ the only jointly invariant measures are easily seen to be Lebesgue measure and the Dirac mass at 0 . The same holds if one takes a polynomial $P(\cdot)$ with integer coefficients and considers $\left\{E_{P(n)} \mid n \in \mathbb{N}\right\}$. Furstenberg asked whether Lebesgue measure is the only nonatomic invariant Borel probability measure for $E_{2}$ and $E_{3}$.

The second example is $M=\operatorname{SL}(n, \mathbb{R}) / \Gamma$ for $n \geq 3$ and a lattice $\Gamma \subset \operatorname{SL}(n, \mathbb{R})$. The Weyl chamber flow is the action of the set $D$ of positive diagonal elements on $M$ by left translation. ( $D$ is isomorphic to $\mathbb{R}^{n-1}$ ).

PROBLEM 17.1. Find all invariant measures for these two examples.

Rudolph [127] showed in 1990 that a measure invariant under both $E_{2}$ and $E_{3}$ for which one of $E_{2}$ and $E_{3}$ has positive entropy is Lebesgue measure. Geometric methods which form the basis of most of the work up to now were introduced in [87]. In 2003 Einsiedler, Katok and Lindenstrauss [31] proved the analogous result for the Weyl chamber flow (assuming positive entropy for one element of the action). See[100] for a survey of the this rapidly developing subject at a recent (but not present) date. 
Fundamentally the issues for the case of positive entropy are reasonably well understood although (possibly formidable) technical problems remain. However, even simple questions remain in the general case. Here is an example.

QUESTION 17.2. Given an Anosov diffeomorphism and a generic ergodic invariant measure (i.e., neither Lebesgue measure nor an atomic one), is there a diffeomorphism that preserves this measure and that is not a power of the Anosov diffeomorphism itself?

Indeed, the zero-entropy case is entirely open, and experts differ on the expected outcome. One can take a geometric or Fourier-analytic approach. The difficulty with the latter one is that even though one has a natural dual available, measures don't behave well with respect to passing to the dual. At the level of invariant distributions there is little difference between rank 1 and higher rank whereas the wealth of invariant measures is quite different between these two situations. The geometric approach produced the results for positive entropy, but in the zero-entropy situation conditional measures on stable and unstable leaves are atomic.

Thus, we either lack the imagination to come up with novel invariant measures or the structure to rule these out.

As to the reason for concentrating on abelian actions, this simply provides the first test case for understanding hyperbolic actions in this respect. Recently, substantial progress was achieved beyond the algebraic or uniformly hyperbolic cases $[75 ; 84]$. The paradigm here is that positive entropy hyperbolic invariant measures are forced to be absolutely continuous if the rank of the action is sufficiently high, e.g., for $\mathbb{Z}^{k}$ actions on $k+1$-dimensional manifolds for $k \geq 2$.

For unipotent actions, by contrast, the Ratner rigidity theory is fairly comprehensive, but here the paradigm is in essence unique ergodicity, which is quite different from the hyperbolic situation.

\section{Rigidity of higher-rank abelian actions (presented by Danijela Damjanovic)}

Consider actions of $A=\mathbb{Z}^{k}$ or $A=\mathbb{R}^{k}$ on a compact manifold, where $k$ is at least 2. One class of such actions consists of $\mathbb{Z}^{k}$-actions on $\mathbb{T}^{N}$ by toral automorphisms; we say that these are genuinely of higher rank if there is a subgroup isomorphic to $\mathbb{Z}^{2}$ that acts by ergodic automorphisms. Another class consists of the action by the diagonal $A=\mathbb{R}^{n-1}$ on $M=\operatorname{SL}(n, \mathbb{R}) / \Gamma$, or actions by a generic hyperplane $\mathbb{R}^{d}$ for $2 \leq d<n-1$. This is a partially hyperbolic action whose neutral direction is the neutral direction for the full Cartan. For the first of these cases Katok and Damjanovic have proved rigidity [24], and this raises the following 
QUESTION 18.1. Can the KAM-methods of Damjanovic and Katok be used to establish rigidity in actions of the second type?

The second situation provides much more geometric structure than the first one, and this can be put to use. Methods of Katok and Spatzier [86] reduce the problem to studying perturbations in the neutral direction, each of which is given by a cocycle over the perturbed action. Therefore the same objective can be achieved by answering the following:

QUESTION 18.2. Can one show cocycle rigidity for the perturbed actions?

Katok and Kononenko [83] have established Hölder cocyle stability for partially hyperbolic diffeomorphisms with the accessibility property that could be put to use here. Progress has been achieved recently by first introducing a new method for proving cocycle rigidity [23] which uses results and methods from algebraic K-theory and then developing this method further so that it applies to cocycle over perturbations and hence produces local rigidity (up to small standard perturbations within the full Cartan subgroup) for the actions of the second kind [25].

\section{Local rigidity of actions (presented by David Fisher)}

For more background, references, more information on the questions raised in this section as well as other interesting questions in this area, the reader should refer to the survey by Fisher in this volume.

DEFINITION 19.1. A homomorphism $i: \Gamma \rightarrow D$ from a finitely generated group $\Gamma$ to a topological group $D$ is said to be locally rigid if any $i^{\prime}$ sufficiently close to $i$ in the compact-open topology is conjugate to $i$ by a small element of $D$.

This is the case for the inclusion of an irreducible cocompact lattice in a semisimple Lie group with no compact or 3-dimensional factors (Calabi-Vesentini, Selberg, Weil).

A basic question posed by Zimmer around 1985 is whether one can do anything of interest if the topological group is the diffeomorphism group of a compact manifold and $\Gamma$ is a lattice in a group $G$ that has no rank-1 factors (for example, $G=\operatorname{SL}(n, \mathbb{R}), G=\operatorname{SL}(n, \mathbb{Z})$ for $n \geq 3$ ). (Katok and collabarators have studied $\Gamma=\mathbb{Z}^{d}$ for $d \geq 2$ with this in view.) Benveniste showed that every isometric action of a cocompact group is locally rigid in $\operatorname{Diff}^{\infty}(M)$, and shortly thereafter Margulis and Fisher showed that any isometric action of a group $\Gamma$ is locally rigid if the group has proprty (T) of Kazhdan, i.e., $H^{1}(\Gamma, \pi)=0$ for every unitary representation $\pi$.

A theorem of Kazhdan asserts that there are cocompact lattices in $S U(1, n)$ that admit nontrivial homomorphisms to $\mathbb{Z}$, and as an easy consequence any 
action of these with connected centralizer has deformations. These deformations are not very interesting, so one can ask.

\section{QUESTION 19.2. Are these the only deformations?}

There are cocompact lattices in $\mathrm{SO}(1, n)$ with embeddings in $\mathrm{SO}(n+1)$, where the resulting action on $S^{n}$ has an infinite-dimensional deformation space. The known deformations do not preserve volume:

QUESTION 19.3. Are there volume-preserving deformations in this situation?

If $\Gamma$ is an irreducible lattice in a semisimple Lie group $G$ that has no compact factors and is contained in a Lie group $H$ with a cocompact lattice $\Lambda$ then $\Gamma$ acts on $H / \Lambda$. In many cases these actions are not locally rigid, such as for lattices in $\mathrm{SU}(1, n)$ and in $\mathrm{SO}(1, n)$.

CONJECTURE 19.4. An action of a lattice $\Gamma$ is locally rigid if there is a map from $G$ onto a group $G_{1}$ that is locally isomorphic to either $S O(1, n)$ or $S U(1, n)$ and acts on a space $X$ in such a way that there is a factorization of $H \Lambda$ to $X$ that intertwines the actions of $\Gamma$ on $H / \Lambda$ and $X$ (the latter induced by $\Gamma \subset G_{1}$.

This is even open for Anosov actions. A simple linear example of these would be the natural action of $\operatorname{SL}(2, \mathbb{Z} \sqrt{2})$ on $\mathbb{T}^{4}$ obtained by the linear action on $\mathbb{R}^{4}$ of the 2 Galois-conjugate embeddings, which have an invariant lattice. In the case of Anosov actions this should be an approachable question. Crossing the preceding example with the identity on a circle should be much harder.

\section{Smooth and geometric rigidity}

CONJECTURE 20.1. A compact negatively curved Riemannian manifold with $C^{1+\text { zygmund }}$ horospheric foliations is locally symmetric.

It is believed that smooth rigidity of systems with smooth invariant foliations should hold with low regularity. Yet this remains an open issue. There is some evidence that this is a hard question. For example, investigations of the Anosov obstruction to $C^{2}$ foliations [61] made clear that its vanishing does not have immediate helpful consequences. And the basic bootstrap [60] does not start at $C^{2}$.

The invariant subbundles $E^{u}$ and $E^{s}$, called the unstable and stable bundles, are always Hölder continuous. For $E^{u}$ this means that there exist $0<\alpha \leq 1$ and $C, \delta>0$ such that $d_{G}\left(E^{u}(p), E^{u}(q)\right) \leq C d_{M}(p, q)^{\alpha}$ whenever $d_{M}(p, q) \leq \delta$, where $d_{G}$ is an appropriate metric on subbundles of $T M$. We say that $E^{u}$ is $C^{\alpha}$ or $\alpha$-Hölder; in case $\alpha=1$ we say $E^{u}$ is $C^{\text {Lip }}$ or Lipschitz continuous. A continuous function $f: U \rightarrow \mathbb{R}$ on an open set $U \subset \mathbb{R}$ is said to be Zygmundregular if there is $K>0$ such that $|f(x+h)+f(x-h)-2 f(x)| \leq K|h|$ for 
all $x \in U$ and sufficiently small $h$. To specify a value of $K$ we may refer to a function as being $K$-Zygmund. The function is said to be "little Zygmund" or $C^{\text {zygmund }}$ if $|f(x+h)+f(x-h)-2 f(x)|=o(|h|)$. Zygmund regularity implies modulus of continuity $O(|x \log | x||)$ and hence $H$-Hölder continuity for all $H<1$ [139, Theorem (3.4)]. It follows from Lipschitz continuity and hence from differentiability. Being "little Zygmund" implies having modulus of continuity $o(|x \log | x||)$. For $r \in \mathbb{N}$ denote by $C^{r, \omega}$ the space of maps whose $r$-th derivatives have modulus of continuity $\omega$. For $r>0$ let $C^{r}=C^{\lfloor r\rfloor, O\left(x^{r-\lfloor r\rfloor}\right)}$.

For the dependence of the leaves on the base point several slightly different definitions are possible. The canonical definition is via the highest possible regularity of lamination charts. One may also look into the transverse regularity of $k$-jets. Alternatively, one can examine the holonomy semigroup, i.e., for pairs of nearby smooth transversals to the lamination one considers the locally defined map between them that is obtained by "following the leaves". By transversality this is well-defined, and for smooth transversals one can discuss the regularity of these maps, which turns out to be largely independent of the transversals chosen. We adopt this notion here and refer to it as the regularity of holonomies or (transverse) regularity of the lamination. There is little difference between these definitions in our context. Following the discussion in [126] we can summarize the relation as follows:

THEOREM 20.2 ([126, Theorem 6.1]). If $r \in \mathbb{R} \cup\{\infty\}, r \notin \mathbb{N} \backslash\{1\}$ then $a$ foliation with uniformly $C^{r}$ leaves and holonomies has $C^{r}$ foliation charts.

However, if $r \in \mathbb{N} \backslash\{1\}$, a foliation with uniformly $C^{r}$ leaves and holonomies need not have $C^{r}$ foliation charts. The problem are mixed partials. Without assuming uniform regularity the above statements can fail drastically: There is a foliation with uniformly $C^{\infty}$ leaves and with (nonuniformly) $C^{\infty}$ holonomies that does not have a $C^{1}$ foliation chart [126, Figure 9]. In our context the regularity is always uniform, so the above result implies that one can define regularity equally well via holonomies or foliation charts. The essential ingredient for Theorem 20.2 is

Theorem 20.3 ([74]). Let $M$ be a $C^{\infty}$ manifold, $F^{u}, F^{s}$ continuous transverse foliations with uniformly smooth leaves, $n \in \mathbb{N}_{0}, \alpha>0, f: M \rightarrow \mathbb{R}$ uniformly $C^{n+\alpha}$ on leaves of $F^{u}$ and $F^{s}$. Then $f$ is $C^{n+\alpha}$.

This leads to the following observation.

THEOREM 20.4. If $r \in \mathbb{R} \cup\{\infty\}, r \notin \mathbb{N} \backslash\{1\}$ and the stable and unstable foliations have uniformly $C^{r}$ holonomies, then there are $C^{r}$ bifoliation charts, i.e., charts that straighten both foliations simultaneously. 
PROOF. By hypothesis every point $p$ has a neighborhood $U$ on which the inverse $[x, y] \mapsto(x, y) \in W_{\varepsilon}^{u}(p) \times W_{\varepsilon}^{s}(p)$ of the local product structure map is uniformly $C^{r}$ in either entry. By Theorem 20.3 it is $C^{r}$.

There is a connection between the regularity of the subbundles and that of the lamination: For any $r \in \mathbb{N} \cup\{\infty\}$ and $\alpha \in[0,1)$ or " $\alpha=$ Lip" a foliation tangent to a $C^{r+\alpha}$ subbundle is itself $C^{r+\alpha}$ [126, Table 1]. (The reverse implication holds only for $r=\infty$ because leaves tangent to a $C^{r}$ subbundle are $C^{r+1}$.)

The invariant subbundles are always Hölder continuous. It should be noted, however, that for $\alpha<1$ the $\alpha$-Hölder condition on subbundles does not imply any regularity of the foliations. Indeed, without a Lipschitz condition even a onedimensional subbundle may not be uniquely integrable, so already continuity of the foliation cannot be obtained this way. On the other hand, there turns out to be a converse connection:

THEOREM 20.5 [63]. If the holonomies are $\alpha$-Hölder and individual leaves are $C^{\infty}$ then the subbundles are $\beta$-Hölder for every $\beta<\alpha$.

There are variants of this for leaves of finite smoothness and almost-everywhere Hölder conditions. Furthermore, whenever bunching-type information gives a particular degree of regularity for the subbundles, one can usually get the same regularity for the holonomies, and vice versa.

CONJECTURE 20.6. If both invariant foliations of an Anosov system are $C^{2}$ then they are both $C^{\infty}$.

Bolder variants of this would replace $C^{2}$ by $C^{1+\text { Lip }}, C^{1+\mathrm{BV}}, C^{1+\text { zygmund }}$ ("little Zygmund") or $C^{1+o(x|\log x|)}$, but the $C^{2}$ version would be spectacular enough, even in the symplectic case.

Note that such rigidity results can only be expected assuming high regularity of both foliations simultaneously because [62] gives a sufficient condition for one foliation to be $C^{2}$ that holds for an open set of dynamical systems.

To prove such results it may be necessary to restrict to the geometric context, where there are extra ingredients that might help. The leaves are spheres, and they are "tied together" by the sphere at infinity (ideal boundary) of the universal cover. An important result by Hamenstädt [58] should help substantially as well:

THEOREM 20.7. If the horospheric foliations are $C^{2}$ then the topological and Liouville entropies of the geodesic flow coincide.

If the Katok entropy conjecture were known this would finish the problem.

Thus, the following problem remains: By exploiting geometric information show that if the horospheric foliations are $C^{2}$ and the topological and Liouville entropies of the geodesic flow coincide then the horospheric foliations are $C^{\infty}$ 
(or $C^{k}$ for sufficiently large $k$ to invoke the bootstrap [60]). This leads to a geometric counterpart of Conjecture 20.6.

CONJECTURE 20.8. A compact negatively curved Riemannian manifold with $C^{2}$ horospheric foliations is locally symmetric.

By [7; 9] this follows from Conjecture 20.6. A related proposal is the following: Give an alternate proof of the Hamenstädt result by showing that if the horospheric foliations are $C^{2}$ then the Jacobian cocycle is cohomologous to a constant (which implies coincidence of Bowen-Margulis measure and Liouville measure, i.e., coincidence of topological and Liouville entropy). The reason that this route is interesting to explore is that it provides a motivation to return to the Anosov cocycle and investigate whether it is at all connected with the Jacobian cocycle in subtle ways.

As noted above, smoothness of invariant structures associated with a hyperbolic dynamical system is necessary for smooth conjugacy to an algebraic model. There are several important instances where such conditions are sufficient.

Smoothness of the invariant foliations of a hyperbolic dynamical system has turned out to be sufficient for smooth conjugacy to an algebraic model in the symplectic case. For geodesic flows even more can be said. Open questions concern the precise amount of smoothness needed and possible conclusions in the absence of symplecticity.

Smoothness of the invariant foliations. The most basic result in this direction is implicit: The proof by Avez [5] that an area-preserving Anosov diffeomorphism of $\mathbb{T}^{2}$ is topologically conjugate to an automorphism actually gives a conjugacy as smooth as the invariant foliations. The definitive result in this setting is worth giving here, because it is suggestive of the work yet to be done in higher dimension.

THEOREM 20.9 [68]. Let $f$ be a $C^{\infty}$ area-preserving Anosov diffeomorphism of $\mathbb{\square}^{2}$. Then the invariant subbundles are differentiable and their first derivatives satisfy the Zygmund condition [139, Section II.3, (3.1)] and hence have modulus of continuity $O(x|\log x|)$ [139, Theorem (3.4)]. There is a cocycle, the Anosov cocycle, which is a coboundary if and only if these derivatives have modulus of continuity $o(x|\log x|)$ or, equivalently, satisfy a "little Zygmund" condition. In this case, or if the derivatives have bounded variation [54], the invariant foliations are $C^{\infty}$ and $f$ is $C^{\infty}$ conjugate to an automorphism.

Note the sharp divide between the general and the smoothly rigid situation. Indeed, the constant defining $O(x|\log x|)$ is nonzero a.e., except when the Anosov cocycle is trivial. Therefore this is the finest possible dichotomy. 
To obtain $C^{\infty}$ foliations it is actually shown first that triviality of the Anosov cocycle implies $C^{3}$ subbundles, and a separate argument then yields $C^{\infty}$ foliations.

Following Guysinsky one can explain the Anosov cocycle using local normal forms. For a smooth area-preserving Anosov diffeomorphism on $\mathbb{T}^{2}$ deLatte [94] showed that one can find local smooth coordinate systems around each point that depend continuously (actually $C^{1}$ ) on the point and bring the diffeomorphism $f$ into the Moser normal form [112]

$$
f(x, y)=\left(\begin{array}{c}
\lambda_{p}^{-1} x / \varphi_{p}(x y) \\
\lambda_{p} y \varphi_{p}(x y)
\end{array}\right)
$$

where $(x, y)$ are in local coordinates around a point $p$ and the expression on the right is in coordinates around $f(p)$. The terms involving $\varphi_{p}$ that depend on the product $x y$ correspond to the natural resonance $\lambda_{p} \lambda_{p}^{-1}=1$ that arises from area-preservation (actually from the family of resonances $\lambda_{p}=\lambda_{p}^{n+1} \lambda_{p}^{-n}$ ). The function $\varphi_{p}$ is as smooth as $f$, and $\varphi_{p}(0)=1$. Now we suppress the (continuous) dependence of $\lambda$ and $\varphi$ on $p$. Note that for a point $(0, y)$ we have

$$
\begin{array}{rc}
D f=\left(\begin{array}{cc}
\lambda^{-1} x y(1 / \varphi)^{\prime}(x y)+\lambda^{-1} / \varphi(x y) & \lambda^{-1} x^{2}(1 / \varphi)^{\prime}(x y) \\
\lambda y^{2} \varphi^{\prime}(x y) & \lambda x y \varphi^{\prime}(x y)+\lambda \varphi(x y)
\end{array}\right) \\
=\left(\begin{array}{cc}
\lambda^{-1} & 0 \\
\lambda y^{2} \varphi^{\prime}(0) & \lambda
\end{array}\right) .
\end{array}
$$

In these local coordinates the unstable direction at a point $(0, y)$ on the stable leaf of $p$ is spanned by a vector $(1, a(y))$. Since this subbundle is invariant under $D f$ and since $f(0, y)=(0, \lambda y)$, the coordinate representation of $D f$ from above gives $a(\lambda y)=\lambda^{2} y^{2} \varphi^{\prime}(0)+\lambda^{2} a(y)$. If the unstable subbundle is $C^{2}$ then differentating this relation twice with respect to $x$ at 0 gives $\lambda^{2} a^{\prime \prime}(0)=$ $2 \lambda^{2} \varphi^{\prime}(0)+\lambda^{2} a^{\prime \prime}(0)$, i.e., $\varphi^{\prime}(0)=0$. This means that the Anosov obstruction is $\varphi^{\prime}(0)$, where $\varphi$ arises from the nonstationary Moser-deLatte normal form. (Thus this is also the obstruction to $C^{1}$ linearization.)

Hurder and Katok verify that $A(p):=\varphi_{p}^{\prime}(0)$ is a cocycle and show that it is nonzero a.e. unless it is null-cohomologous. (Guysinsky's result that $C^{1+B V} \Rightarrow$ $C^{\infty}$ follows because bounded variation implies differentiability almost everywhere.)

The work by Hurder and Katok is actually carried out for the weak subbundles of volume-preserving Anosov flows on three-manifolds. In this situation analogous issues arise relative to the strong subbundles. These can be worked out with closely related techniques:

THEOREM 20.10 [46]. Let $M$ be a 3-manifold, $\varphi: \mathbb{R} \times M \rightarrow M$ a $C^{k}$ volumepreserving Anosov flow. Then $E^{u} \oplus E^{s}$ is Zygmund-regular, and there is an 
obstruction to higher regularity that can be described geometrically as the curvature of the image of a transversal under a return map. This obstruction defines the cohomology class of a cocycle, and the following are equivalent:

(1) The longitudinal KAM-cocycle is a coboundary.

(2) $E^{u} \oplus E^{s}$ is "little Zygmund".

(3) $E^{u} \oplus E^{s}$ is Lipschitz.

(4) $E^{u} \oplus E^{s} \in C^{k-1}$.

(5) $\varphi$ is a suspension or contact flow.

\section{PROBLEM 20.11. Extend this result to higher dimension.}

The complications in higher dimension are due in large part to the simple fact that when the invariant foliations are not one-dimensional there may be different contraction and expansion rates at any given point. Therefore a first step in working on this problem would be to assume uniform quasiconformality in stable and unstable directions. This has strong structural implications in itself, though (Theorem 16.6, [32; 76; 128]).

Different contraction and expansion rates are responsible already for the fact that in higher dimension the transverse regularity is usually lower than in the two-dimensional case. Note that the results there never assert higher regularity for both foliations than in the two-dimensional area-preserving case. If the obstruction vanishes that was used to show optimality of those results, then the regularity "jumps" up a little, and a further obstruction, associated with different contraction and expansion rates, may prohibit regularity $C^{1+O(x|\log x|)}$. Only when all those finitely many obstructions vanish can we have $C^{1+O(x|\log x|)}$. These obstructions are best described in normal form [55], as is the Anosov cocycle.

To give a sample we show that a "1-2-resonance" produces an obstruction to $C^{1}$ foliations. To work with the simplest possible situation consider a 3dimensional Anosov diffeomorphism $f$ with fixed point $p$ such that the eigenvalues $0<\lambda<\mu<1<\eta<\infty$ of $D f_{p}$ satisfy $\mu=\lambda \eta$. (This is a variant of the 1-2-resonance $\lambda_{1}=\lambda_{2}^{2}$ for a symplectic system.) Up to higherorder terms that might arise from higher resonances the normal form of $f$ at $p$ is $f(x, y, z)=(\eta x, \mu y+a x z, \lambda z)$. Representing $E^{u}$ along the $z$-axis by $\left(1, v_{1}(z), v_{2}(z)\right.$ gives

$$
D f_{(0,0, z)}\left(1, v_{1}(z), v_{2}(z)\right)=\left(\eta, a z+\mu v_{1}(z), \lambda v_{2}(z)\right),
$$

which rescales to $\left(1, a z / \eta+\mu v_{1}(z) / \eta, \lambda v_{2}(z) / \eta\right)$. Invariance of $E^{u}$ therefore yields

$$
v_{1}(\lambda z)=a z / \eta+\mu v_{1}(z) / \eta
$$


Differentiating twice with respect to $z$ gives $\lambda v_{1}^{\prime}(0)=a / \eta+(\mu / \eta) v_{1}^{\prime}(0)$, which implies $a=0$ since $\lambda=\mu / \eta$. Thus the resonance term $a$ in the normal form is an obstruction to $C^{1}$ Anosov splitting. (One can verify this without using normal forms, but the calculation is somewhat longer.) By the way, the work of Kanai mentioned in the next subsection made a rather stringent curvature pinching assumption to rule out a number of low resonances. The refinements by Feres and Katok that led to an almost complete proof of Theorem 20.17 centered on a careful study of the resonances that might arise without such pinching assumptions. This was delicate work because the issue are not only resonances at periodic points, but "almost resonances" between Lyapunov exponents. The papers [39; 37] contain an impressive development of these ideas.

While there is an analog of the Anosov cocycle in higher dimension, its vanishing is known to be necessary only for $C^{2}$ foliations [61] and is not known to lead to higher regularity of the invariant foliations. Thus it has not yielded any effective application, and the central portion of the above approach falls apart.

The bootstrap to $C^{\infty}$ subbundles works in full generality, even without areapreservation, although it usually starts at regularity higher than $C^{3}$ (see [60; 47]). In other words, once the invariant foliations have a sufficiently high degree of regularity, they are always $C^{\infty}$.

Smooth rigidity. The main issue in higher dimension is to conclude from smoothness of the invariant foliations that there is a smooth conjugacy to an algebraic model, and to identify the right algebraic model in the first place.

A result that appeared after systematic development of the continuous time situation (see also [7, Theorem 3]) will serve to illustrate this:

THEOREM 20.12 ([8]). Let $M$ be a $C^{\infty}$ manifold with an $C^{\infty}$ affine connection $\nabla, f: M \rightarrow M$ a topologically transitive Anosov diffeomorphism preserving $\nabla$ with $E^{u}, E^{s} \in C^{\infty}$. Then $f$ is $C^{\infty}$ conjugate to an automorphism of an infranilmanifold. The invariant connection hypothesis can be replaced by invariance of a smooth symplectic form.

Note the absence of a topological hypothesis. (There is a finite-smoothness sharpening of this result [38] that does not use the powerful theorem of Gromov central to the proof by Benoist and Labourie.)

Now we turn to the continuous time case, where these developments are most significant.

The history begins with the work of Ghys [51], who classified volume-preserving Anosov flows on 3-manifolds with smooth invariant foliations into three types: suspensions of hyperbolic automorphisms of the torus, geodesic flows on surfaces of constant negative curvature (up to finite coverings), and a new type of flow that differs from the old ones by a special time change. If the flow 
is known to be geodesic then the smooth conjugacy to the constant curvature geodesic flow preserves topological and measure-theoretic entropies, and hence by entropy rigidity (see page 310 and [79]) the original metric is constantly curved. The work towards classification of flows with smooth invariant foliations has followed this model closely. Before describing this, let us mention in passing the secondary issue of reducing the regularity at which the classification becomes possible. In the situation of Ghys one can use an analysis of 3-dimensional volume-preserving Anosov flows and a result entirely analogous to Theorem 20.9 [68] to conclude:

THEOREM 20.13 [51;68]. A negatively curved metric on a compact surface is hyperbolic if its horocycle foliations are $C^{1+o(x|\log x|)}$.

In higher dimension the seminal work is due to Kanai [78]. He was the first to implement the following strategy: If one assumes that the invariant foliations are smooth then one can study Lie bracket relations between the stable and unstable subbundles. The interaction between these and the dynamics can be used to build an invariant connection (named after him now [91]) and to show that it is flat, which in turn is used to build a Lie algebra structure that is identifiable as a standard model.

He obtained the following result:

THEOREM 20.14 [78]. The geodesic flow of a strictly 9/4-pinched negatively curved Riemannian metric on a compact manifold is smoothly conjugate to the geodesic flow of a hyperbolic manifold if the invariant foliations are $C^{\infty}$.

Two groups picked up this lead, with the primary aim of removing the pinching hypothesis, which in particular rules out nonconstantly curved locally symmetric spaces as models. It also emerged that the main import of the assumptions is dynamical rather than geometric, and that therefore one should look for theorems about flows more general than geodesic ones.

Feres and Katok [39; 37] built on Kanai's idea by refining his arguments with intricate analyses of resonance cases for Lyapunov exponents to cover most of the ground in terms of the admissible algebraic models.

THEOREM 20.15 [37]. Consider a compact Riemannian manifold $M$ of negative sectional curvature. Suppose the horospheric foliations are smooth. If the metric is 1/4-pinched or $M$ has odd dimension then the geodesic flow is smoothly conjugate to that of a hyperbolic manifold. If the dimension is 2 (mod 4) then the geodesic flow is smoothly conjugate to that of a quotient of complex hyperbolic space.

Some of the results proved along the way to this conclusion did not assume that the flow under consideration is geodesic. The refinements over Kanai's work 
were, in the case of the first hypothesis, a more delicate argument for vanishing of the curvature of the Kanai connection. Under the second hypothesis Feres shows that if the Kanai connection is not flat then the invariant subbundles split further (resonance considerations enter here), and a connection associated with this further splitting must be locally homogeneous.

Roughly simultaneously the complete result about smooth conjugacy was obtained by Benoist, Foulon and Labourie [7]. Not only does it include all geodesic flows, but it requires only a contact structure, which turned out to require substantial additional work. This makes it a proper counterpart of the three-dimensional result of Ghys:

THEOREM 20.16 [7]. Suppose $\Phi$ is a contact Anosov flow on a compact manifold of dimension greater than 3, with $C^{\infty}$ Anosov splitting. Then there is an essentially unique time change and a finite cover on which the flow is $C^{\infty}$ conjugate to the geodesic flow of a negatively curved manifold.

What enables the authors to give a monolithic proof (as opposed to covering the various classes of symmetric spaces one by one) is a rigidity result by Gromov $[53 ; 6 ; 138]$. This is the place where substantial regularity is needed, and on an $m$-dimensional manifold one can replace $C^{\infty}$ in hypothesis and conclusion by $C^{k}$ with $k \geq m^{2}+m+2$. This theorem is invoked in the first major step of the proof, to produce a homogeneous structure: The diffeomorphisms of the universal cover that respect the splitting and the flow form a Lie group that acts transitively. (Gromov's theorem produces this structure on an open dense set, and the Kanai connection is used to extend it.) Step two determines the structure of this group and its Lie algebra, and step three develops the dynamics of the group and relates it to the expected algebraic model.

The Feres-Katok approach needs a slightly different minimal regularity. In fact, if one adds the a posteriori redundant assumption of (nonstrict) $\frac{1}{4}$-pinching (or merely strict $\frac{4}{25}$-pinching) then $C^{5}$ horospheric foliations always force rigidity [60].

We note an amplified version for the case of geodesic flows in which the conjugacy conclusion for geodesic flows is replaced by isometry of the metrics due to a more recent rigidity result by Besson, Courtois and Gallot, Theorem 20.29.

THEOREM 20.17. If the horospheric foliations of a negatively curved compact Riemannian manifold are $C^{\infty}$ then the metric is locally symmetric (up to isometry).

The above result subsumes several classification steps. First of all, one obtains an orbit equivalence, which implies coincidence of the Lyapunov cocycles (periodic data). But furthermore, the original result in [7] directly arrives at a smooth conjugacy, which means that periods of periodic orbits are preserved as well. 
This is an extra collection of moduli for the continuous time case. Finally, in the case of geodesic flows, there is, in addition, the Besson-Courtois-Gallot Theorem 20.29, which gives the isometry.

While the regularity of the invariant subbundles is usually substantially lower than in the two-dimensional case, it is widely believed that the minimal regularity for such smooth rigidity results should be $C^{2}$ or even $C^{1+\text { Lip }}$, i.e., quite close to that in Theorem 20.9. Indeed, these foliations are hardly ever $C^{1+\text { Lip: }}$

THEOREM 20.18. For an open dense set of symplectic Anosov systems the regularity predicted by computing $B^{u}$ only from periodic points is not exceeded (i.e., if the rates compare badly at a single periodic point then the regularity is correspondingly low - at that periodic point) [62]. An open dense set of Riemannian metrics do not have $C^{1+\text { Lip }}$ horospheric foliations [62].

Furthermore, for any $\varepsilon>0$ there is an open set of symplectic Anosov diffeomorphisms for which the subbundles and holonomies are $C^{\varepsilon}$ at most on a (Lebesgue) null set [63].

If the invariant subbundles are $C^{2}$ then the Liouville measure coincides with the Bowen-Margulis measure of maximal entropy [58; 91]. (For Finsler metrics this is false [117].) According to the Katok Entropy Rigidity Conjecture (page 310), this should imply that the manifold is locally symmetric. Optimists might suspect that rigidity already appears from $C^{1+o(x|\log x|)}$ or $C^{1+\text { zygmund }}$ on, but there is no evidence to that effect (save for Theorem 20.18).

Another result of Ursula Hamenstädt is worth remarking on here. It says that for contact Anosov flows with $C^{1}$ invariant foliations fixing the time parametrization fixes all other moduli of smooth conjugacy.

THEOREM 20.19 [57]. If two conjugate (not just orbit equivalent) Anosov flows both have $C^{1}$ Anosov splitting and preserve a $C^{2}$ contact form then the conjugacy is $C^{2}$.

The $C^{1}$ assumption on the splitting is not vacuous, but not stringent either, being satisfied by an open set of systems. Note that the conjugacy preserves both Lebesgue and Bowen-Margulis measure. If one keeps in mind that smooth conjugacy has been established mainly with one side being algebraic, this result is striking in its generality.

Inasmuch as they refer to flows, the hypotheses of the preceding rigidity results do not distinguish between the regularity of the strong versus weak invariant foliations. The reason is that for geodesic flows strong and weak foliations have the same regularity due to the invariant contact structure: The strong subbundles are obtained from the weak ones by intersecting with the kernel of the smooth canonical contact form. 
Plante [121] showed that the strong foliations may persistently fail to be $C^{1}$, namely when the asymptotic cycle of volume measure is nonzero. Even though the latter is not the case for (noncontact) perturbations of geodesic flows, these flows may still fail to have $C^{1}$ strong foliations (see [116; 11], where the contact form is "twisted" by an extra "magnetic force term", which does not produce a nontrivial asymptotic cycle).

Entropy rigidity. A different rigidity conjecture was put forward by Katok in a paper that proved it for surfaces [79].

The result that prompted the conjecture is

THEOREM 20.20 [79]. For the geodesic flow of a unit-area Riemannian metric without focal points on a surface of negative Euler characteristic E the Liouville and topological entropies lie on either side of $\sqrt{-2 \pi E}$, with equality (on either side) only for constantly curved metrics.

CONJECTURE 20.21 [79, p. 347]. Liouville measure has maximal entropy only for locally symmetric metrics, i.e., only in these cases do the topological and Liouville entropies agree.

This says that equivalence of Bowen-Margulis and Lebesgue measure only occurs for locally symmetric spaces. This conjecture has engendered an enormous amount of activity and remains unresolved. The exact nature of the results in [79] suggests some variants of this conjecture, however, that have been adressed more successfully.

THEOREM 20.22 [44]. The conjecture holds locally along one-parameter perturbations of constantly curved metrics, but in dimension 3 it is no longer the case that a hyperbolic metric (with unit volume) maximizes Liouville entropy.

The Katok entropy rigidity conjecture cannot take quite so neat a form as it does for surfaces. Foulon notes that for flows in dimension three it extends beyond the realm of geodesic flows:

THEOREM 20.23 [45]. A smooth contact Anosov flow on a three-manifold whose topological and Liouville entropies coincide is, up to finite covers, conjugate to the geodesic flow of a constantly curved compact surface.

Conjecture 20.24 (Foulon). Three-dimensional $C^{\infty}$ Anosov flows for which Bowen-Margulis and Lebesgue measure are equivalent must be $C^{\infty}$ conjugate to either a suspension of a toral automorphism or the geodesic flow of a compact hyperbolic surface.

That a metric is locally symmetric has been proved under a stronger but suggestive hypothesis [97]. Consider the universal cover $M$ of the manifold in question and for each $x \in M$ define a measure $\lambda_{x}$ on the sphere at infinity by 
projecting the Lebesgue measure on the sphere $S_{x} M$ along geodesics starting at $x$ (Lebesgue or visibility measure). Use a construction of the (Bowen-)Margulis measure [104] to define measures $v_{x}$ on the sphere at infinity [56].

THEOREM 20.25. If there is a constant a such that $\lambda_{x}=a v_{x}$ for all $x$ then $M$ is symmetric.

Proof. By [96; 137] it is asymptotically harmonic, and by [47] and Theorem 20.29 below it is symmetric.

In fact, one can also define a harmonic measure $\eta_{x}$ at infinity for every $x \in M$ by using Brownian motion.

THEOREM 20.26 [95; 81]. In the case of surfaces the harmonic measure class coincides with the Lebesgue class only when the curvature is constant.

Conjecture 20.27 (The "Sullivan Conjecture”, [131, p. 724]). In higher dimension the coincidence of the harmonic and visibility measure classes happens only for locally symmetric spaces.

THEOREM 20.28. If any two of these three measures here defined are proportional for every $x$ then $M$ is symmetric.

PROOF. This again follows from [96; 137; 47; 9].

The goal can be restated as the requirement to relax the hypothesis from proportionality to mutual absolute continuity [97].

Coming from rather a different direction, Besson, Courtois and Gallot found themselves addressing a related issue by showing that topological entropy is minimized only by locally symmetric metrics. Strictly speaking, their result concerns the volume growth entropy $h$ of a compact Riemannian manifold, which is the exponential growth rate of the volume of a ball in the universal cover as a function of the radius. This is a lower bound for the topological entropy of the geodesic flow with equality if the sectional curvature is nonpositive [103] (in fact, when there are no conjugate points [50]).

THEOREM 20.29 ([9]). Let $X, Y$ be compact oriented connected $n$-dimensional manifolds, $f: Y \rightarrow X$ continuous of nonzero degree. If $g_{0}$ is a negatively curved locally symmetric metric on $X$ then every metric $g$ on $Y$ satisfies $h^{n}(Y, g) \operatorname{Vol}(Y, g) \geq|\operatorname{deg}(f)| h^{n}\left(X, g_{0}\right) \operatorname{Vol}\left(X, g_{0}\right)$ and for $n \geq 3$ equality occurs iff $(Y, g)$ is locally symmetric (of the same type as $\left(X, g_{0}\right)$ ) and $f$ is homotopic to a homothetic covering $(Y, g) \rightarrow\left(X, g_{0}\right)$. In particular, locally symmetric spaces minimize entropy when the volume is prescribed.

A version of this result holds for nonpositively curved locally symmetric spaces of rank 1, and one may ask whether their method extends to higher rank. 
A complementary result, about leaving the realm of geodesic flows, is contained in the work [118] of the brothers Paternain: "Twisting" any Anosov geodesic flow (by adding a "magnetic" term to the Hamiltonian) strictly decreases topological entropy.

\section{Quantitative symplectic geometry (Helmut Hofer)}

Denote by Symp $^{2 n}$ the category of $2 n$-dimensional symplectic manifolds with embeddings serving as the morphisms. This carries the action of $(0, \infty)$ by rescaling: $\alpha^{*}(M, \omega)=(M, \alpha \omega)$. Consider a subcategory $\mathfrak{C}$ that is invariant under this action and $[0, \infty]$ with the standard ordering (on which one has the same action). We do not require it to be a full subcategory.

DEFINITION 21.1. A (generalized) symplectic capacity for $\mathfrak{C}$ is an equivariant functor $c: \mathfrak{C} \rightarrow[0, \infty]$ with the property that $c((M, \omega))>0$ if $M \neq \varnothing$. For $1 \leq d \leq n$ a $d$-capacity is a capacity such that $0<c\left(B^{2 d} \times \mathbb{R}^{2 n-2 d}\right)<\infty$ and $c\left(B^{2 d-2} \times \mathbb{R}^{2 n-2 d+2}\right)=\infty$, where $B^{d}$ denotes the open unit ball in $\mathbb{R}^{d}$.

An example of a $n$-capacity is

$$
c(M, \omega):=\left(\int_{M} \omega^{n}\right)^{1 / n} .
$$

Let $B^{2 n}(a)$ denote the ball of radius $(a / \pi)^{1 / 2}$ and define $Z^{2 n}(a)=B^{2}(a) \times$ $\mathbb{R}^{2 n-2}$ We put $B^{2 d}=B^{2 d}(1)$ and similarly $Z^{2 d}=Z^{2 d}(1)$. Gromov's nonsqueezing result implies the existence of 1-capacities. If $B^{2 n}(a)$ symplectically embeds into $Z^{2 n}(b)$ then $a \leq b$. Therefore one can take

$$
c_{B^{2 n}}(M, \omega):=\sup \left\{a \mid\left(B^{2 n}, a \omega\right) \text { symplectically embeds into }(M, \omega)\right\}
$$

or

$$
c^{Z^{2 n}}(M, \omega):=\inf \left\{a \mid(M, \omega) \text { symplectically embeds into }\left(Z^{2 n}, a \omega\right)\right\} .
$$

There are many 1-capacities one can construct from Floer theory, GromovWitten theory, symplectic field theory or contact homology, and many questions in symplectic geometry can be answered by constructing such a functor. No example of a $d$-capacity for $1<d<n$ is known. Therefore it is important to ask

QUESTION 21.2. Are there $d$-capacities for $d$ other than 1 and $n$ in dimension $2 n$, where $n \geq 3$ ?

This is a fundamental question about the nature of symplectic geometry. Clearly the $n$-capacities are volume-related invariants and 1-capacities are invariants of a 2-dimensional kind related to 2-dimensional cross-sections. The essence of Question 21.2 is captured by the next question concerned with dimension six. 
QUESTION 21.3. Is there an $\varepsilon>0$ such that for every $r>0$ there is a symplectic embedding of $B^{2}(\varepsilon) \times B^{4}(r)$ into $B^{4}(1) \times \mathbb{R}^{2}$ ?

If the answer is "no" it means that in dimension six a 2-capacity exists, and in this case it is very likely that a proof has to be based on some "new symplectic technology".

The next question is exploring the problem if in some sense the technology to deal with symplectic geometry in dimension four is complete. Given a positive quadratic form $Q$ define $E_{Q}:=\{Q<1\} \subset \mathbb{R}^{2 n}$ to be the associated ellipsoid. Then there is a unique $a \in \Sigma:=\left\{a \in(0, \infty)^{n} \mid a_{1} \leq a_{2} \leq \ldots \leq a_{n}\right\}$ such that $E$ is by a linear symplectic map the same as the ellipsoid $E(a):=\left\{x=\left(z_{1}, \ldots, z_{n}\right) \in\right.$ $\left.\left.\mathbb{R}^{2 n}\left|\sum\right| z_{i}\right|^{2} / a_{i}<1\right\}$.

On $\Sigma$ define a "linear" partial ordering $\leq_{l}$ by $a \leq_{l} b$ : $\Longleftrightarrow$ there exists a linear symplectic map $T$ such that $T(E(a)) \subset E(b)$. By some linear algebra this order structure is the same as requiring $a_{i} \leq b_{i}$ for all $i$. Define a "nonlinear" partial ordering $\leq_{n l}$ by $a \leq_{n l} b: \Longleftrightarrow$ there is a symplectic embedding of $E(a)$ into $E(b)$. It is a nontrivial result (due to Ekeland and Hofer) that on the set of points "between" $(1, \ldots, 1)$ and $(2, \ldots, 2)$ these two orderings are the same, but this fails on any larger set (Lalonde and McDuff for $n=2$, Schlenk in general).

Consider capacities on ellipsoids and for $a \in \Sigma$ order the numbers $\left\{j a_{i} \mid j \in\right.$ $\mathbb{N}, i=1, \ldots, n\}$ by size with multiplicities and denote this sequence by $c_{k}(a)$ (If $a=(1,5)$ we get $12345567891010 \ldots$ ). These are capacities for each $k$ (and are due to Ekeland and Hofer).

QUESTION 21.4. Is $a \leq_{n l} b$ in $\mathbb{R}^{4}$ equivalent to $c_{k}(a) \leq c_{k}(b)$ for all $k$ and $a_{1} a_{2} \leq b_{1} b_{2}$ (this is the volume constraint)?

If the answer is indeed "yes" a proof can be expected to be very hard. Particular cases of this question are:

QUESTION 21.5. Is $(1,8) \leq_{n l}(3,3)$ ? Is $(1,4) \leq_{n l}(2,2)$ ?

Schlenk can symplectically embed

$$
E(1,8) \rightarrow E(3.612,3.612) \text { and } E(1,4) \rightarrow E(2.692,2.692) .
$$

Observe that $E(1,4)$ and $E(2,2)$ have the same volume, so an embedding will be very tight.

In general, consider a symplectic category $\mathfrak{C}$ and denote the collection of capacities on it by $S$. Then one can generate new ones. Consider a function $f:[0, \infty]^{n} \rightarrow[0, \infty]$ with $f(1, \ldots, 1)>0$ that is positively 1 -homogeneous $(f(t a)=t f(a))$ and monotone (if $a_{i} \leq b_{i}$ then $\left.f(a) \leq f(b)\right)$. Then, given capacities $c_{1}, \ldots, c_{n} \in S$ we get a new capacity $f\left(c_{1}, \ldots, c_{n}\right)$. Also, if $c_{k} \rightarrow c$ as $k \rightarrow \infty$ and $c\left(B^{2 n}\right)>0$ then $c$ is a capacity as well. The following is essentially a rephrasing of Question 21.4. 
QUESTION 21.6. Do the $c_{k}$ defined above together with $\left(\int \omega^{2}\right)^{1 / 2}$ generate $S$ in this way?

\section{Hilbert's sixteenth problem (presented by Yulij Ilyashenko)}

Question 22.1 (Hilbert's 16Th Problem). What can be said about the number and location of the limit cycles of a polynomial ordinary differential equation in the plane?

This has been among the most persistent in Hilbert's list, and therefore even simplified versions make for substantial problems:

QUESTION 22.2 (HILBERT'S 16TH PROBLEM FOR QUADRATIC POLYNOMIALS). What can be said about the number and location of the limit cycles of an ordinary differential equation in the plane whose right-hand side is a quadratic polynomial vector field?

This question remains unresolved as well. There are partial results by Ilyashenko and Llibre of the following type. For a Zariski-open set of quadratic vector fields one can define a numerical characteristic of each vector field and then bound the number of limit cycles in terms of this parameter.

Numerous related problems may be found in the survey [69]

\section{Foliations (presented by Steven Hurder)}

Consider a compact manifold $M$ with a foliation $F$.

QUESTION 23.1. Can a leaf $L$ in a minimal set $Z \subset M$ of the foliation be deformed? Or can the minimal set be deformed?

Reeb showed that if there is a compact leaf with trivial holonomy (i.e., only the identity) then it has a foliated neighborhood that is a product, i.e., the situation is far from rigid. On the other hand, results by Stowe show that if there is enough cohomology data then one cannot move the leaf.

QUESTION 23.2. If $Z$ is a minimal set in $M$, are all leaves in $Z$ diffeomorphic up to covers?

Duminy proved in 1982 that if the foliation is $C^{2}$ with codimension 1 leaves and $Z$ is exceptional (i.e., neither $M$ nor a single leaf) then all leaves in $Z$ have Cantor ends. 


\section{4. "Fat" self-similar sets (Mark Pollicott)}

A similarity on $\mathbb{R}^{d}$ is a map $T: \mathbb{R}^{d} \rightarrow \mathbb{R}^{d}$ for which $\|T(x, y)-T(u, v)\|=$ $r\|(x, y)-(u, v)\|$, where $0<r<1$ and $\|\cdot\|$ is the Euclidean norm. Given similarities $T_{1}, \ldots, T_{n}: \mathbb{R}^{d} \rightarrow \mathbb{R}^{d}$ a set $\Lambda$ is said to be self-similar if $\Lambda=$ $\bigcup_{i=1}^{n} T_{i}(\Lambda)$. One may ask how "big" such sets can be, for example, how close to $d$ the Hausdorff dimension can be, whether they can have positive Lebesgue measure or open interior.

In the case that $d=2$, there are examples of self-similar sets with empty interior and positive Lebesgue measure (this is due to Csörnyei, Jordan, Pollicott, Preiss and Solomyak [22] and answers a question of Peres and Solomyak [119]). The construction uses 10 contractions (all by a factor of 3), but there is some latitude in how the similarities are chosen, and a different construction accomplishes the same result using 6 similarities.

QUESTION 24.1. Can one find examples using fewer similarities?

It is interesting to note that there are apparently no analogous results when $d=1$.

QUESTION 24.2. Are the examples of self similar sets with positive measure but empty interior in $\mathbb{R}$ ?

Easier results are obtained from Sierpinski triangles. If $1 / 2<\lambda<1$ the similarities

$$
\begin{aligned}
& T_{0}(x, y)=(\lambda x, \lambda y)+(0,0) \\
& T_{1}(x, y)=(\lambda x, \lambda y)+(1 / 2,0) \\
& T_{2}(x, y)=(\lambda x, \lambda y)+(0,1 / 2)
\end{aligned}
$$

produce "fat" Sierpinski triangles $\Lambda_{\lambda}$ (the case $\lambda=1 / 2$ gives the standard Sierpinski triangle).

It is easy to check that when $0<\lambda<1 / 2$ one obtains a Cantor set with $\operatorname{dim}_{H}\left(\Lambda_{\lambda}\right)=-\log 3 / \log \lambda$.

THEOREM 24.3 (JORDAN [72]).

$$
\operatorname{dim}_{H}\left(\Lambda_{\lambda}\right)=-\frac{\log 3}{\log \lambda} \quad \text { for a.e. } \lambda \in\left[1 / 2,(4 / 3)^{1 / 3}\right] .
$$

There is a dense set $D \subset[1 / 2,1 / \sqrt{3}]$ such that $\operatorname{dim}_{H}\left(\Lambda_{\lambda}\right)<-\log 3 / \log \lambda$ for $\lambda \in D$. One of the most interesting remaining questions is the following.

QUESTION 24.4. How large is the exceptional set D? Is it uncountable? Does it have nonzero Hausdorff dimension?

The next result was proved in [73] by Jordan and Pollicott, and in [15] by Broomhead, Montaldi and Sidorov. Let $m$ be the $d$-dimensional Lebesgue measure. 
THEOREM 24.5. $m\left(\Lambda_{\lambda}\right)>0$ for a.e. $\lambda \in[0.585 \ldots, 0.647 \ldots]$ and $\operatorname{int}\left(\Lambda_{\lambda}\right) \neq \varnothing$ for $\lambda>.647 \ldots$

This suggests two natural questions.

QUESTION 24.6. What is the largest value of $\lambda$ such that $\Lambda_{\lambda}$ has empty interior?

Sidorov conjectures that the correct value is the reciprocal of the golden ratio.

QUESTION 24.7. Is there any $\lambda \in[0.585 \ldots, 0.647 \ldots]$ for which $\operatorname{int}\left(\Lambda_{\lambda}\right)=\varnothing$ and $m\left(\Lambda_{\lambda}\right)>0$ ?

\section{References}

[1] Dmitri Viktorovich Anosov: Geodesic flows on Riemann manifolds with negative curvature, Proc. Steklov Inst. Math. 90 (1967); Roughness of geodesic flows on compact Riemannian manifolds of negative curvature, Dokl. Akad. Nauk SSSR 145 (1962), 707-709; Ergodic properties of geodesic flows on closed Riemannian manifolds of negative curvature, Dokl. Akad. Nauk SSSR 151 (1963), 1250-1252.

[2] Dmitri Viktorovich Anosov and Anatole B. Katok: New examples in smooth ergodic theory: Ergodic diffeomorphisms, Trans. Moscow Math. Soc. 23 (1970), 1-35.

[3] Dmitriĭ Viktorovich Anosov and Yakov Sinai: Some smooth ergodic systems, Russian Math. Surveys 22 (1967), no. 5, 103-167.

[4] Zvi Artstein and Alexander Vigodner: Singularly perturbed ordinary differential equations with dynamic limits, Proc. Roy. Soc. Edinburgh Sect. A 126 (1996), 541569.

[5] André Avez: Anosov diffeomorphisms, Proceedings of the Topological Dynamics Symposium (Fort Collins, CO, 1967), 17-51, W. Gottschalk and J. Auslander, eds., Benjamin, New York, 1968.

[6] Yves Benoist: Orbites des structures rigides (d'après M. Gromov), Integrable systems and foliations/Feuilletages at systèmes intégrables (Montpellier, 1995), 117, Progr. Math. 145, Birkhäuser, Boston, 1997.

[7] Yves Benoist, Patrick Foulon, and François Labourie: Flots d'Anosov à distributions stable et instable différentiables, J. Amer. Math. Soc. 5 (1992), no. 1, 33-74.

[8] Yves Benoist and François Labourie: Sur les difféomorphismes d'Anosov affins à feuilletages stable et instables différentiables, Invent. Math. 111 (1993), no. 2, 285308.

[9] Gérard Besson, Gilles Courtois, and Sylvestre Gallot: Entropies et rigidités des espaces localement symétriques de courbure strictement négative, Geom. Funct. Anal. 5 (1995), no. 5, 731-799; Minimal entropy and Mostow's rigidity theorems, Ergodic Theory Dynam. Systems 16 (1996), no. 4, 623-649.

[10] M. Blank and L. Bunimovich: Multicomponent dynamical systems: SRB measures and phase transitions, Nonlinearity 16 (2003), no. 1, 387-401. 
[11] Jeffrey Boland: On the regularity of the Anosov splitting and conjugacy types for magnetic field flows, unpublished.

[12] M. Boyle and T. Downarowicz: The entropy theory of symbolic extensions, Invent. Math. 156 (2004), 119-161.

[13] M. Boyle and T. Downarowicz: Symbolic extension entropy: $C^{r}$ examples, products and flows, Discrete Contin. Dynam. Systems 16 (2006), no. 2, 329-341.

[14] M. Boyle, D. Fiebig and U. Fiebig: Residual entropy, conditional entropy and subshift covers, Forum Math. 14 (2002), 713-757.

[15] D. Broomhead, J. Montaldi, and N. Sidorov: Golden gaskets: Variations on the Sierpiński sieve, Nonlinearity 17 (2004), 1455-1480.

[16] Leonid A. Bunimovich, Nicolai I. Chernov, and Yakov G. Sinai: Statistical properties of two-dimensional hyperbolic billiards, Russian Math. Surveys 46 (1991), $47-106$.

[17] Keith Burns, Federico Rodríguez Hertz, Jana Rodríguez Hertz, Anna Talitskaya, and Raúl Ures: Density of accessibility for partially hyperbolic diffeomorphisms with one dimensional center, in preparation.

[18] Keith Burns and Amie Wilkinson: On the ergodicity of partially hyperbolic systems, preprint, 2005.

[19] J. Buzzi: Intrinsic ergodicity of smooth interval maps, Israel J. Math. 100 (1997), $125-161$.

[20] Kariane Calta: Veech surfaces and complete periodicity in genus two, J. Amer. Math. Soc. 17 (2004), no. 4, 871-908.

[21] Gonzalo Contreras and Gabriel Paternain: Genericity of geodesic flows with positive topological entropy on $S^{2}$, J. Differential Geom. 61 (2002), no. 1, 1-49.

[22] M. Csörnyei, T. Jordan, M. Pollicott, D. Preiss, and B. Solomyak: Positive measure self-similar sets without interior, Ergodic Theory Dynam. Systems 26 (2006), no. 3, 755-758.

[23] Danijela Damjanovic and Anatole Katok: Periodic cycle functionals and cocycle rigidity for certain partially hyperbolic $\mathbb{R}^{k}$-actions, Discrete Contin. Dynam. Systems 13 (2005), 985-1005.

[24] Danijela Damjanovic and Anatole Katok: Local rigidity of partially hyperbolic actions of $\mathbb{Z}^{k}$ and $\mathbb{R}^{k}, k \geq 2$, I: KAM method and actions on the torus, preprint. http://www.math.psu.edu/katok_a/papers.html/

[25] Danijela Damjanovic and Anatole Katok: Local rigidity of partially hyperbolic actions, II: Restrictions of Weyl chamber flows on $\operatorname{SL}(n, \mathbb{R}) / \Gamma$, preprint. http:// www.math.psu.edu/katok_a/papers.html/

[26] D. Dolgopyat and A. Wilkinson: Stable accessibility is $C^{1}$ dense, Geometric methods in dynamics, II, Astérisque 287 (2003), xvii, 33-60.

[27] T. Downarowicz: Entropy of a symbolic extension of a totally disconnected dynamical system, Ergodic Theory Dynam. Systems 21 (2001), 1051-1070. 
[28] T. Downarowicz: Entropy Structure, J. Anal. Math. 96 (2005), 57-116.

[29] T. Downarowicz and J. Serafin: Possible entropy functions, Israel J. Math. 135 (2003), 221-251.

[30] T. Downarowicz and S. Newhouse: Symbolic extensions in smooth dynamical systems, Invent. Math. 160 (2005), no. 3, 453-499.

[31] Manfred Einsiedler, Elon Lindenstrauss, and Anatole Katok: Invariant measures and the set of exceptions to Littlewood's conjecture, Ann. of Math. (2) 164 (2006), no. 2, 513-560.

[32] Yong Fang: Smooth rigidity of uniformly quasiconformal Anosov flows. Ergodic Theory Dynam. Systems 24 (2004), no. 6, 1937-1959.

[33] Bassam Fayad and Raphaël Krikorian: manuscript in preparation.

[34] Bassam Fayad and Anatole B. Katok: Constructions in elliptic dynamics, Herman memorial issue, Ergodic Theory Dynam. Systems 24 (2004), no. 5, 1477-1520.

[35] Bassam Fayad and M. Saprykina: Weak mixing disc and annulus diffeomorphisms with arbitrary Liouville rotation number on the boundary, Ann. Sci. Ècole Norm. Sup. (4) 38 (2005), no. 3, 339-364.

[36] Bassam Fayad, M. Saprykina, and Alistair Windsor: manuscript in preparation.

[37] Renato Feres: Geodesic flows on manifolds of negative curvature with smooth horospheric foliations, Ergodic Theory Dynam. Systems 11 (1991), no. 4, 653-686.

[38] Renato Feres: The invariant connection of a $\frac{1}{2}$-pinched Anosov diffeomorphism and rigidity, Pacific J. Math. 171 (1995), no. 1, 139-155.

[39] Renato Feres and Anatole Katok: Invariant tensor fields of dynamical systems with pinched Lyapunov exponents and rigidity of geodesic flows, Ergodic Theory Dynam. Systems 9 (1989), no. 3, 427-432; Anosov flows with smooth foliations and rigidity of geodesic flows on three-dimensional manifolds of negative curvature, Ergodic Theory Dynam. Systems 10 (1990), no. 4, 657-670.

[40] M. Field, I. Melbourne, and A. Török: Stable ergodicity for smooth compact Lie group extensions of hyperbolic basic sets, Ergodic Theory Dynam. Systems 25 (2005), 517-551.

[41] M. J. Field, I. Melbourne, and A. Török: Stability of mixing and rapid mixing for hyperbolic flows, Ann. of Math. (2), to appear.

[42] Todd Fisher: The topology of hyperbolic attractors on compact surfaces, Ergodic Theory Dynam. Systems 26 (2006), no. 5, 1511-1520.

[43] Todd Fisher: Hyperbolic sets that are not locally maximal, Ergodic Theory Dynam. Systems 26 (2006), no. 5, 1491-1509.

[44] Livio Flaminio: Local entropy rigidity for hyperbolic manifolds, Comm. Anal. Geom. 3 (1995), no. 3-4, 555-596.

[45] Patrick Foulon: Entropy rigidity of Anosov flows in dimension three, Ergodic Theory Dynam. Systems 21 (2001), no. 4, 1101-1112. 
[46] Patrick Foulon and Boris Hasselblatt: Zygmund strong foliations, Israel J. Math. 138 (2003), 157-169.

[47] Patrick Foulon and François Labourie: Sur les variétés compactes asymptotiquement harmoniques, Invent. Math. 109 (1992), no. 1, 97-111.

[48] John Franks: Anosov diffeomorphisms, Global analysis, 61-93, Proc. Sympos. Pure Math. 14, American Mathematical Society, Providence, RI, 1970.

[49] John Franks and Robert Williams: Anomalous Anosov flows, Global theory of dynamical systems, 158-174, Zbigniew Nitecki and R. Clark Robinson, eds., Lecture Notes in Mathematics 819, Springer, Berlin, 1980.

[50] A. Freire and Ricardo Mañé: On the entropy of the geodesic flow in manifolds without conjugate points, Invent. Math. 69 (1982), no. 3, 375-392.

[51] Etienne Ghys: Déformations de flots d'Anosov et de groupes Fuchsiens, Ann. Inst. Fourier (Grenoble) 42 (1992) no. 1-2, 209-247.

[52] Matthew Grayson, Charles C. Pugh, and Michael Shub: Stably ergodic diffeomorphisms, Ann. of Math. (2) 140 (1994), no. 2, 295-329.

[53] Michael Gromov: Rigid transformations groups, Géometrie differentielle (Paris, 1986), 65-139, Daniel Bernard and Yvonne Choquet-Bruhat, eds., Travaux en Cours 33, Hermann, Paris, 1988,

[54] Misha Guysinsky: Smoothness of holonomy maps derived from unstable foliation, Smooth ergodic theory and its applications (Seattle, WA, 1999), 785-790, Proc. Sympos. Pure Math. 69, American Mathematical Society, Providence, RI, 2001.

[55] Misha Guysinsky and Anatole Katok: Normal forms and invariant geometric structures for dynamical systems with invariant contracting foliations, Math. Res. Lett. 5 (1998), no. 1-2, 149-163.

[56] Ursula Hamenstädt: A new description of the Bowen-Margulis measure, Ergodic Theory Dynam. Systems 9 (1989), no. 3, 455-464; Boris Hasselblatt: A new construction of the Margulis measure for Anosov flows, Ergodic Theory Dynam. Systems 9 (1989), no. 3, 465-468.

[57] Ursula Hamenstädt: Regularity of time-preserving conjugacies for contact Anosov flows with $C^{1}$ Anosov splitting, Ergodic Theory Dynam. Systems 13 (1993), no. 1, $65-72$.

[58] Ursula Hamenstädt: Invariant two-forms for geodesic flows, Math. Ann. 301 (1995), no. 4, 677-698.

[59] Michael Handel and William Thurston: Anosov flows on new three manifolds, Invent. Math. 59 (1980), no. 2, 95-103.

[60] Boris Hasselblatt: Bootstrapping regularity of the Anosov splitting, Proc. Amer. Math. Soc. 115, (1992), no. 3, 817-819.

[61] Boris Hasselblatt: Anosov obstructions in higher dimension, Internat. J. Math. 4 (1993), no. 3, 395-407. 
[62] Boris Hasselblatt: Regularity of the Anosov splitting and of horospheric foliations, Ergodic Theory Dynam. Systems 14 (1994), no. 4, 645-666.

[63] Boris Hasselblatt and Amie Wilkinson: Prevalence of non-Lipschitz Anosov foliations, Ergodic Theory Dynam. Systems 19 (1999), no. 3, 643-656.

[64] Federico Rodríguez Hertz: Global rigidity of certain abelian actions by toral automorphisms, in preparation.

[65] Federico Rodríguez Hertz, Jana Rodríguez Hertz, and Raúl Ures: Acessibility and stable ergodicity for partially hyperbolic diffeomorphisms with $1 D$ center bundle, preprint, 2005.

[66] Eberhard Hopf: Statistik der geodätischen Linien in Mannigfaltigkeiten negativer Krümmung, Berichte über die Verhandlungen der Sächsischen Akademie der Wissenschaften zu Leipzig, Mathematisch-Physikalische Klasse 91 (1939), 261-304.

[67] Pascal Hubert and T. A. Schmidt: An introduction to Veech surfaces, Handbook of dynamical systems, 1B, 501-526, Elsevier, Amsterdam, 2006.

[68] Steven Hurder and Anatole Katok: Differentiability, rigidity, and Godbillon-Vey classes for Anosov flows, Publ. Math. IHES 72 (1990), 5-61.

[69] Y. Ilyashenko: Centennial history of Hilbert's 16th problem, Bull. Amer. Math. Soc. 39, (2002), no. 3, 301-354.

[70] Michael V. Jakobson: Absolutely continuous invariant measures for one-parameter families of one-dimensional maps, Comm. Math. Phys. 81 (1981), no. 1, 39 88.

[71] Esa Järvenpää and Tapani Tolonen: Relations between natural and observable measures, Nonlinearity 18 (2005), no. 2, 897-912.

[72] Thomas Jordan: Dimension of fat Sierpinski gaskets, Real Anal. Exchange 31 (2005/06), no. 1, 97-110.

[73] Thomas Jordan and Mark Pollicott: Properties of measures supported on fat Sierpinski carpets, Ergodic Theory Dynam. Systems 26 (2006), no. 3, 739-754.

[74] Jean-Lin Journé: A regularity lemma for functions of several variables, Rev. Mat. Iberoamericana 4 (1988), no. 2, 187-193.

[75] Boris Kalinin and Anatole B. Katok: Measure rigidity beyond uniform hyperbolicity: Invariant measures for Cartan actions on tori, J. Mod. Dyn. 1 (2007), no. 1, 123-146. http://www.math.psu.edu/katok_a/papers.html/

[76] Boris Kalinin and Victoria Sadovskaya: On local and global rigidity of quasiconformal Anosov diffeomorphisms, J. Inst. Math. Jussieu 2 (2003), no. 4, 567-582.

[77] Boris Kalinin and Ralf Spatzier: Rigidity of the measurable structure for algebraic actions of higher-rank abelian groups, Ergodic Theory Dynam. Systems 25 (2005), no. $1,175-200$.

[78] Masahiko Kanai: Geodesic flows of negatively curved manifolds with smooth stable and unstable foliations, Ergodic Theory Dynam. Systems 8 (1988), no. 2, 215-239. 
[79] Anatole B. Katok: Entropy and closed geodesics, Ergodic Theory Dynam. Systems 2 (1982), no. 2, 339-367.

[80] Anatole B. Katok: The growth rate for the number of singular and periodic orbits for a polygonal billiard, Comm. Math. Phys. 111 (1987), 151-160.

[81] Anatole B. Katok: Four applications of conformal equivalence to geometry and dynamics, Charles Conley memorial issue, Ergodic Theory Dynam. Systems 8* (1988), 139-152.

[82] Anatole B. Katok and Boris Hasselblatt: Introduction to the modern theory of dynamical systems, Cambridge University Press, Cambridge, 1995.

[83] Anatole B. Katok and Alexey Kononenko: Cocycle stability for partially hyperbolic systems, Math. Res. Lett. 3 (1996), 191-210.

[84] Anatole Katok and Federico Rodríguez Hertz: Uniqueness of large invariant measures for $Z^{k}$ actions with Cartan homotopy data, J. Mod. Dyn. 1 (2007), no. 2 , 287-300. http://www.math.psu.edu/katok_a/papers.html/

[85] Anatole B. Katok and Ralf Jürgen Spatzier: First cohomology of Anosov actions of higher rank abelian groups and applications to rigidity, Publ. Math. IHES 79 (1994), 131-156.

[86] Anatole B. Katok and Ralf Jürgen Spatzier: Subelliptic estimates of polynomial differential operators and applications to rigidity of abelian actions, Math. Res. Lett. 1 (1994), 193-202.

[87] Anatole B. Katok and Ralf Jürgen Spatzier: Invariant measures for higher rank hyperbolic abelian actions, Ergodic Theory Dynam. Systems 16 (1996), 751-778.

[88] Steven Kerckhoff, Howard Masur, and John Smillie: Ergodicity of billiard flows and quadratic differentials. Ann. of Math. (2) 124 (1986), no. 2, 293-311; A rational billiard flow is uniquely ergodic in almost every direction, Bull. Amer. Math. Soc. (N. S.) 13 (1985), no. 2, 141-142.

[89] Yuri Kifer: Averaging principle for fully coupled dynamical systems and large deviations, Ergodic Theory Dynam. Systems 24 (2004), 847-871.

[90] Yuri Kifer: Some recent advances in averaging, Modern dynamical systems and applications, 385-403, Cambridge University Press, Cambridge, 2004.

[91] Gerhard Knieper: Hyperbolic dynamics and Riemannian geometry. Handbook of dynamical systems, 1A, 453-545, Boris Hasselblatt and Anatole Katok, eds., Elsevier, Amsterdam, 2002.

[92] Gerhard Knieper and Howard Weiss: $C^{\infty}$ genericity of positive topological entropy for geodesic flows on $S^{2}$, J. Differential Geom. 62 (2002), no. 1, 127-141.

[93] J. Kulesza: Zero-dimensional covers of finite-dimensional dynamical systems, Ergodic Theory Dynam. Systems 15 (1995), 939-950.

[94] David DeLatte: Nonstationary normal forms and cocycle invariants, Random Comput. Dynam. 1 (1992/93), no. 2, 229-259; On normal forms in Hamiltonian 
dynamics, a new approach to some convergence questions, Ergodic Theory Dynam. Systems 15 (1995), no. 1, 49-66.

[95] François Ledrappier: Propriété de Poisson et courbure négative, C. R. Acad. Sci. Paris Sér. I Math. 305 (1987), no. 5, 191-194.

[96] François Ledrappier: Harmonic measures and Bowen-Margulis measures, Israel J. Math. 71 (1990), no. 3, 275-287.

[97] François Ledrappier: Applications of dynamics to compact manifolds of negative curvature, Proceedings of the International Congress of Mathematicians, 1, 2 (Zürich, 1994), 1195-1202, Birkhäuser, Basel, 1995.

[98] Douglas Lind and Jean-Paul Thouvenot: Measure-preserving homeomorphisms of the torus represent all finite entropy ergodic transformations, Math. Systems Theory 11 (1977/78), no. 3, 275-282.

[99] E. Lindenstrauss: Mean dimension, small entropy factors and an embedding theorem, Publ. Math. IHES 89 (1999), 227-262.

[100] Elon Lindenstrauss: Rigidity of multiparameter actions, Probability in mathematics, Israel J. Math. 149 (2005), 199-226.

[101] E. Lindenstrauss and B. Weiss: Mean topological dimension, Israel J. Math 115 (2000), 1-24.

[102] Anthony Manning: There are no new Anosov diffeomorphisms on tori, Amer. J. Math. 96 (1974), 422-429.

[103] Anthony Manning: Topological entropy for geodesic flows, Ann. of Math. (2) 110 (1979), no. 3, 567-573.

[104] Grigorĭi A. Margulis: Certain measures that are connected with $\mathrm{Y}$-flows on compact manifolds (in Russian), Funkcional. Anal. i Priložen. 4 (1970), no. 1, 62-76; English transl. in Functional Anal. Appl. 4 (1970), 55-67.

[105] Howard Masur: the growth rate of trajectories of a quadratic differential, Ergodic Theory Dynam. Systems 10 (1990), 151-176.

[106] Curtis T. McMullen: Dynamics of $\operatorname{SL}(2, \mathbb{R})$ over moduli spaces in genus two, preprint.

[107] M. Misiurewicz: On non-continuity of topological entropy, Bull. Acad. Polon. Sci. Sér. Sci. Math. Astronom. Phys. 19 (1971), 319-320.

[108] M. Misiurewicz: Diffeomorphism without any measure with maximal entropy, Bull. Acad. Polon. Sci. Sér. Sci. Math. Astronom. Phys. 21 (1973), 903-910.

[109] M. Misiurewicz: Topological conditional entropy, Studia Math. 55 (1976), 175200.

[110] Michał Misiurewicz: Ergodic natural measures, Algebraic and topological dynamics, 1-6, S. Kolyada, Y. Manin, and T. Ward, eds., Contemp. Math. 385, American Mathematical Society, Providence, RI, 2005. 
[111] M. Misiurewicz and A. Zdunik: Convergence of images of certain measures, Statistical physics and dynamical systems (Köszeg, 1984), 203-219, Progr. Phys. 10, Birkhäuser, Boston, 1985.

[112] Jürgen K. Moser: The analytic invariants of an area-preserving mapping near a hyperbolic fixed point, Comm. Pure Appl. Math. 9 (1956), 673-692.

[113] Sheldon Newhouse: On codimension one Anosov diffeomorphisms, Amer. J. Math. 92 (1970), 761-770.

[114] Viorel Niţică and Andrei Török: An open dense set of stably ergodic diffeomorphisms in a neighborhood of a non-ergodic one, Topology 40 (2001), no. 2, 259-278.

[115] Jacob Palis and Jean-Christophe Yoccoz: Rigidity of centralizers of diffeomorphisms. Ann. Sci. École Norm. Sup. (4) 22 (1998), no. 1, 81-98.

[116] Gabriel Pedro Paternain: On the regularity of the Anosov splitting for twisted geodesic flows, Math. Res. Lett. 4 (1997), no. 6, 871-888.

[117] Gabriel Pedro Paternain: On two noteworthy deformations of negatively curved Riemannian metrics, Discrete Contin. Dynam. Systems 5 (1999), no. 3, 639-650.

[118] Gabriel Pedro Paternain and Miguel Paternain: Anosov geodesic flows and twisted symplectic structures, International Conference on Dynamical Systems (Montevideo, 1995), 132-145, Longman, Harlow, 1996; First derivative of topological entropy for Anosov geodesic flows in the presence of magnetic fields, Nonlinearity 10 (1997), no. $1,121-131$.

[119] Y. Peres and B. Solomyak: Problems on self-similar and self-affine maps: An update, Progr. Probab. 46 (2000), 95-106.

[120] Yakov B. Pesin: Characteristic Ljapunov exponents, and smooth ergodic theory (in Russian), Uspehi Mat. Nauk 32 (1977), no. 4 (196), 55-112, 287; English translation in Russian Math. Surveys 32 (1977), no. 4, 55-114.

[121] Joseph F. Plante: Anosov flows, Amer. J. Math. 94 (1972), 729-754.

[122] M. Pollicott: On the rate of mixing of Axiom A flows, Invent. Math. 81 (1985), 427-447.

[123] Charles C. Pugh and Michael Shub: Stable ergodicity and partial hyperbolicity, International Conference on Dynamical Systems (Montevideo, 1995), 182-187, Longman, Harlow, 1996; Stably ergodic dynamical systems and partial hyperbolicity, J. Complexity 13 (1997), no. 1, 125-179.

[124] Charles C. Pugh and Michael Shub: Stably ergodic dynamical systems and partial hyperbolicity, J. Complexity 13 (1997), no. 1, 125-179.

[125] Charles C. Pugh and Michael Shub: Stable ergodicity and julienne quasi-conformality, J. Eur. Math. Soc. (JEMS) 2 (2000), no. 1, 1-52.

[126] Charles C. Pugh, Michael Shub and Amie Wilkinson: Hölder foliations, Duke Math. J. 86 (1997), no. 3, 517-546.

[127] Daniel Rudolph: $\times 2$ and $\times 3$ invariant measures and entropy, Ergodic Theory Dynam. Systems 10 (1990), no. 2, 395-406. 
[128] Victoria Sadovskaya: On uniformly quasiconformal Anosov systems, Math. Res. Lett. 12 (2005), 425-441.

[129] Richard Evan Schwartz: Obtuse triangular billiards, II: 100 Degrees worth of periodic billiard trajectories, preprint, 2005 http://www.math.brown.edu/-res/Papers/ deg100.pdf. (See also Obtuse triangular billiards, I: Near the (2, 3, 6) triangle, Experiment. Math. 15 (2006), no. 2, 161-182.)

[130] Steven Smale: Mathematical problems for the next century, Mathematics: Frontiers and perspectives, 271-294, American Mathematical Society, Providence, RI, 2000.

[131] Dennis Sullivan: The Dirichlet problem at infinity for a negatively curved manifold, J. Differential Geom. 18 (1983), no. 4, 723-732.

[132] Domokos Szász and Tamás Varjú: Local limit theorem for the Lorentz process and its recurrence in the plane, Ergodic Theory Dynam. Systems. 24 (2004), 257278.

[133] Yoshio Togawa: Centralizers of $C^{1}$-diffeomorphisms, Proc. Amer. Math. Soc. 71 (1978), no. 2, 289-293.

[134] Yaroslav B. Vorobets: Ergodicity of billiards in polygons (in Russian) Mat. Sb. 188 (1997), no. 3, 65-112; English translation in Sb. Math. 188 (1997), no. 3, 389434.

[135] Lai-Sang Young: Ergodic theory of differentiable dynamical systems, Real and complex dynamical systems: Proceedings of the NATO Advanced Study Institute (Hillerød, 1993), 293-336, Bodil Branner and Paul Hjorth, eds., NATO Adv. Sci. Inst. Ser. C Math. Phys. Sci. 464, Kluwer, Dordrecht, 1995.

[136] Lai Sang Young: Statistical properties of dynamical systems with some hyperbolicity, Ann. of Math. (2) 147 (1998), 558-650.

[137] Cheng Bo Yue: On the Sullivan conjecture, Random Comput. Dynam. 1 (1992), no. 2, 131-145.

[138] Abdelghani Zeghib: On Gromov's theory of rigid transformation groups: A dual approach, Ergodic Theory Dynam. Systems 20 (2000), no. 3, 935-946; Sur les groupes de transformations rigides: théorème de l'orbite dense-ouverte, pp. 169188 in Rigidité, groupe fondamental et dynamique, Panor. Synthèses 13, Soc. Math. France, Paris, 2002.

[139] Antoni Szczepan Zygmund: Trigonometric series, Cambridge University Press, Cambridge, 1959 (and 1968, 1979, 1988), revised version of Trigonometrical series, Monografje Matematyczne 5, Z subwencji Funduszu kultury narodowej, Warszawa, 1935.

BORIS HASSELBLATT

DEPARTMENT OF MATHEMATICS

TUFTS UNIVERSITY

MEDFORD, MA 02155

Boris.Hasselblatt@Tufts.edu 